\title{
COMPLEX NEUTROSOPHIC SUBSEMIGROUPS AND IDEALS
}

\author{
MUHAMMAD GULISTAN ${ }^{1, *}$, ASGHAR KHAN ${ }^{2}$, AMIR ABDULLAH ${ }^{1}$, NAVEED \\ YAQOOB $^{3}$ \\ ${ }^{1}$ Department of Mathematics, Hazara University, Mansehra, Pakistan \\ ${ }^{2}$ Department of Mathematics, Abdul Wali Khan University Mardan, Mardan, Pakistan \\ ${ }^{3}$ Department of Mathematics, College of Science Al-Zulfi, Majmaah University, Al-Zulfi, Saudi Arabia \\ *Corresponding author: azhar4set@yahoo.com
}

\begin{abstract}
In this article we study the idea of complex neutrosophic subsemigroups. We define the Cartesian product of complex neutrosophic subsemigroups, give some examples and study some of its related results. We also define complex neutrosophic (left, right, interior) ideal in semigroup. Furthermore, we introduce the concept of characteristic function of complex neutrosophic sets, direct product of complex neutrosophic sets and study some results prove on its.
\end{abstract}

\section{IntroduCtion}

In 1965, Zadeh, ( [1]) presented the idea of a fuzzy set. Atanassov in 1986, ( [2]) initiated the notion of intuitionistic fuzzy set, which is the generalization of a fuzzy set. Neutrosophic set was first proposed by Smarandache in 1999 ( [5]), which is the generalization of a fuzzy set and intuitionistic fuzzy set. Neutrosophic set is characterized by a truth membership function, an indeterminacy membership function and a falsity membership function. It must be noted that there are lots of researchers that worked at complex number and fuzzy sets, for instance Buckly ( [6]), Nguyen et al. ( [7]) and Zhang et al. ( [10]). On the other hand Ramot

Received $19^{\text {th }}$ September, 2017; accepted $5^{\text {th }}$ December, 2017; published $3^{\text {rd }}$ January, 2018.

2010 Mathematics Subject Classification. 03B52.

Key words and phrases. complex fuzzy sets; complex neutrosophic sets; fuzzy subsemigroups; complex neutrosophic subsemigroups; complex neutrosophic ideals.

(C) 2018 Authors retain the copyrights of their papers, and all open access articles are distributed under the terms of the Creative Commons Attribution License. 
et al. ( $[8])$ presented a innovative come close to that is entirely unlike from other researchers, wherever they extensive the variety of membership function to unit circle in the complex plane, unlike the others who limited to. Further to solve enigma they added an extra terms which is called phase term in translating human language to complex valued functions on physical terms and vice versa (for more information, see ( [8]). Abd Uazeez et al. in 2012 ( [12]), added the non-membership term to the idea of complex fuzzy set which is known as complex intuitionistic fuzzy sets, the range of values are extended to the unit circle in complex plan for both membership and non-membership functions instead of [0, 1]. In 2016, Mumtaz Ali et al. ( [14]), more extended the concept of complex fuzzy set, complex intuitionistic fuzzy set, and introduced the concept of complex neutrosophic sets, which is a collection of a complex truth membership function, a complex indeterminacy membership function and a complex falsity membership function. The idea of a fuzzy set in the model of semigroups was first initiated by Kuroki in 1979 ( [3]), and defined fuzzy subsemigroups. Vildan and Halis in 2017 ( [15]), extended the concept of fuzzy subgroups on the base of neutrosophic sets, which is known as neutrosophic subgroups .

Due to the motivation and inspiration of the above discussion. In this paper we are initiating the study of complex neutrosophic semigroups. This paper introduce the notion of complex neutrosophic subsemigroups and Cartesian product of complex neutrosophic subsemigroups with the help of example. We define characteristic function of complex neutrosophic set, direct product of complex neutrosophic sets, complex neutrosophic ideals (left, right, interior) and proved some results.

\section{Preliminaries}

Here in this part we gathered some basic helping materials.

Definition 2.1. ([1]) A function $f$ is defined from a universe $\mathcal{X}$ to a closed interval [0,1] is called a fuzzy set, i.e., a mapping:

$$
f: \mathcal{X} \longrightarrow[0,1]
$$

Definition 2.2. ( [8]) A complex fuzzy set (CFS) $\mathcal{C}$ over the universe $\mathcal{X}$, is defined an object having of the form:

$$
\mathcal{C}=\left\{\left(x, \mu_{\mathcal{C}}(x)\right): x \in \mathcal{X}\right\}
$$

where $\mu_{\mathcal{C}}(x)=r_{\mathcal{C}}(x) \cdot e^{i \omega_{\mathcal{C}}(x)}$, here the amplitude term $r_{\mathcal{C}}(x)$ and phase term $\omega_{\mathcal{C}}(x)$, are real valued functions, for every $x \in \mathcal{X}$, the amplitude term $\mu_{\mathcal{C}}(x): \mathcal{X} \rightarrow[0,1]$ and phase term $\omega_{\mathcal{C}}(x)$ lying in the interval $[0,2 \pi]$.

Definition 2.3. ([13]) Let $\mathcal{C}_{1}$ and $\mathcal{C}_{2}$ be any two complex Atanassov's intuitionistic fuzzy sets (CAIFSs) over the universe $\mathcal{X}$, where

$$
\mathcal{C}_{1}=\left\{\left\langle x, r_{\mathcal{C}_{1}}(x) \cdot e^{i \nu_{\mathcal{C}_{1}}}(x), k_{\mathcal{C}_{1}}(x) \cdot e^{i \omega_{\mathcal{C}_{1}}(x)}\right\rangle: x \in \mathcal{X}\right\}
$$


and

$$
\mathcal{C}_{2}=\left\{\left\langle x, r_{\mathcal{C}_{2}}(x) \cdot e^{i \nu_{\mathcal{C}_{2}}(x)}, k_{\mathcal{C}_{2}}(x) \cdot e^{i \omega_{\mathcal{C}_{2}}(x)}\right\rangle: x \in \mathcal{X}\right\}
$$

Then

\section{Containment:}

$$
\begin{gathered}
\mathcal{C}_{1} \subseteq \mathcal{C}_{2} \Leftrightarrow r_{\mathcal{C}_{1}}(x) \leq r_{\mathcal{C}_{2}}(x), k_{\mathcal{C}_{1}}(x) \geq k_{\mathcal{C}_{2}}(x) \text { and } \\
\nu_{\mathcal{C}_{1}}(x) \leq \nu_{\mathcal{C}_{2}}(x), \omega_{\mathcal{C}_{1}}(x) \geq \omega_{\mathcal{C}_{2}}(x)
\end{gathered}
$$

\section{Equal:}

$$
\begin{gathered}
\mathcal{C}_{1}=\mathcal{C}_{2} \Leftrightarrow r_{\mathcal{C}_{1}}(x)=r_{\mathcal{C}_{2}}(x), k_{\mathcal{C}_{1}}(x)=k_{\mathcal{C}_{2}}(x) \text { and } \\
\nu_{\mathcal{C}_{1}}(x)=\nu_{\mathcal{C}_{2}}(x), \omega_{\mathcal{C}_{1}}(x)=\omega_{\mathcal{C}_{2}}(x)
\end{gathered}
$$

Definition 2.4. ([14]) Let $\mathcal{X}$ be a universe of discourse, and $x \in \mathcal{X}$. A complex neutrosophic set (CNS) $\mathcal{C}$ in $\mathcal{X}$ is characterized by a complex truth membership function $\mathcal{C}_{T}(x)=p_{\mathcal{C}}(x) \cdot e^{i \mu_{\mathcal{C}}(x)}$, a complex indeterminacy membership function $\mathcal{C}_{I}(x)=q_{\mathcal{C}}(x) \cdot e^{i \nu_{\mathcal{C}}(x)}$ and a complex falsity membership function $\mathcal{C}_{F}(x)=r_{\mathcal{C}}(x) \cdot e^{i \omega_{\mathcal{C}}(x)}$. The values $\mathcal{C}_{T}(x), \mathcal{C}_{I}(x), \mathcal{C}_{F}(x)$ may lies all within the unit circle in the complex plane, where $p_{\mathcal{C}}(x)$, qC $(x)$, $r_{\mathcal{C}}(x)$ and $\mu_{\mathcal{C}}(x), \nu_{\mathcal{C}}(x) \omega_{\mathcal{C}}(x)$ are amplitude terms and phase terms, respectively, and where $p_{\mathcal{C}}(x), q_{\mathcal{C}}(x)$, $r_{\mathcal{C}}(x) \in[0,1]$, such that, $0 \leq p_{\mathcal{C}}(x)+q_{\mathcal{C}}(x)+r_{\mathcal{C}}(x) \leq 3$ and $\mu_{\mathcal{C}}(x), \nu_{\mathcal{C}}(x) \omega_{\mathcal{C}}(x) \in[0,2 \pi]$.

The complex neutrosophic set can be represented in the form as:

$$
\mathcal{C}=\left\{\left\langle\begin{array}{c}
x, \mathcal{C}_{T}(x)=p_{\mathcal{C}}(x) \cdot e^{i \mu_{\mathcal{C}}(x)}, \mathcal{C}_{I}(x)=q_{\mathcal{C}}(x) \cdot e^{i \nu_{\mathcal{C}}(x)}, \\
\mathcal{C}_{F}(x)=r_{\mathcal{C}}(x) \cdot e^{i \omega_{\mathcal{C}}(x)}
\end{array}\right): x \in \mathcal{X}\right\} .
$$

Example 2.1. Let $\mathcal{X}=\left\{x_{1}, x_{2}, x_{3}\right\}$ be the universe set and $\mathcal{C}$ be a complex neutrosophic set which is given by:

$$
\mathcal{C}=\left\{\begin{array}{c}
\left\langle x_{1}, 0.2 e^{0.5 \pi i}, 0.3 e^{0.6 \pi i}, 0.4 e^{0.8 \pi i}\right\rangle,\left\langle x_{2}, 0.4 e^{0.6 \pi i}, 0.5 e^{1.3 \pi i}, 0.1 e^{0.6 \pi i}\right\rangle \\
\left\langle x_{3}, 0.1 e^{0.6 \pi i}, 0.3 e^{0.9 \pi i}, 0.9 e^{0.7 \pi i}\right\rangle
\end{array}\right\}
$$

Definition 2.5. ([3]) A fuzzy subset $\mathcal{A}$ of a semigroup $\mathcal{S}$ is said to be a fuzzy subsemigroup of $\mathcal{S}$ if its satisfy the following condition:

$$
\mathcal{A}(x \cdot y) \geq \mathcal{A}(x) \wedge \mathcal{A}(y) \forall x, y \in \mathcal{S}
$$

Definition 2.6. ([15]) Let $\mathcal{G}$ be any group with multiplication and $\mathcal{N}$ be a neutrosophic set on $\mathcal{G}$. Then $\mathcal{N}$ is said to be a neutrosophic subgroup (NSG) of $\mathcal{G}$, if its satisfy the following conditions:

$(\mathrm{NSG} 1): \mathcal{N}(x \cdot y) \geq \mathcal{N}(x) \wedge \mathcal{N}(y)$, i.e.

$T_{\mathcal{N}}(x \cdot y) \geq T_{\mathcal{N}}(x) \wedge T_{\mathcal{N}}(y), I_{\mathcal{N}}(x \cdot y) \geq I_{\mathcal{N}}(x) \wedge I_{\mathcal{N}}(y)$ and $F_{\mathcal{N}}(x \cdot y) \leq F_{\mathcal{N}}(x) \vee F_{\mathcal{N}}(y)$.

$(\mathrm{NSG} 2): \mathcal{N}\left(x^{-1}\right) \geq \mathcal{N}(x)$, i.e., $T_{\mathcal{N}}\left(x^{-1}\right) \geq T_{\mathcal{N}}(x), I_{\mathcal{N}}\left(x^{-1}\right) \geq I_{\mathcal{N}}(x)$ and $F_{\mathcal{N}}\left(x^{-1}\right) \leq F_{\mathcal{N}}(x)$, for all $x$ and $y$ in $\mathcal{G}$. 
Lemma 2.1. ( [16]) For a semigroup $\mathcal{S}$, the following conditions are equivalent.

(1) $\mathcal{S}$ is regular.

(2) $\mathcal{R} \cap \mathcal{L}=\mathcal{R} \mathcal{L}$ for every right ideal $\mathcal{R}$ of $\mathcal{S}$ and every left ideal $\mathcal{L}$ of $\mathcal{S}$.

\section{Complex Neutrosophic Subsemigroup}

Note: It should be noted that through out in this part we use a capital letter $\mathcal{C}$ to denote a complex neutrosophic set;

$$
\mathcal{C}=\left\{\left\langle T_{\mathcal{C}}=p_{\mathcal{C}} \cdot e^{i \mu_{\mathcal{C}}}, I_{\mathcal{C}}=q_{\mathcal{C}} \cdot e^{i \nu_{\mathcal{C}}}, F_{\mathcal{C}}=r_{\mathcal{C}} \cdot e^{i \omega_{\mathcal{C}}}\right\rangle\right\}
$$

Definition 3.1. A complex neutrosophic set $\mathcal{C}=\left\{\left\langle T_{\mathcal{C}}=p_{\mathcal{C}} \cdot e^{i \mu_{\mathcal{C}}}, I_{\mathcal{C}}=q_{\mathcal{C}} \cdot e^{i \nu_{\mathcal{C}}}, F_{\mathcal{C}}=r_{\mathcal{C}} \cdot e^{i \omega_{\mathcal{C}}}\right\rangle\right\}$ on a semigroup $\mathcal{S}$ is known as a complex neutrosophc subsemigroup (CNSG), if its satisfy the following condition:

$\mathcal{C}(x y) \geq \min \{\mathcal{C}(x), \mathcal{C}(y)\}$ i.e.,

(i) $p_{\mathcal{C}}(x y) \cdot e^{i \mu_{\mathcal{C}}(x y)} \geq \min \left\{p_{\mathcal{C}}(x) \cdot e^{i \mu_{\mathcal{C}}(x)}, p_{\mathcal{C}}(y) \cdot e^{i \mu_{\mathcal{C}}(y)}\right\}$

(ii) $q_{\mathcal{C}}(x y) \cdot e^{i \nu_{\mathcal{C}}(x y)} \geq \min \left\{q_{\mathcal{C}}(x) \cdot e^{i \nu_{\mathcal{C}}(x)}, q_{\mathcal{C}}(y) \cdot e^{i \nu_{\mathcal{C}}(y)}\right\}$

(iii) $r_{\mathcal{C}}(x y) \cdot e^{i \omega_{\mathcal{C}}(x y)} \leq \max \left\{r_{\mathcal{C}}(x) \cdot e^{i \omega_{\mathcal{C}}(x)}, r_{\mathcal{C}}(y) \cdot e^{i \omega_{\mathcal{C}}(y)}\right\}, \forall x, y \in \mathcal{S}$.

Example 3.1. Let $\mathcal{S}=\{1,2,3\}$ be a semigroup with the following multiplication table:

\begin{tabular}{|l|l|l|l|}
\hline$\cdot$ & 1 & 2 & 3 \\
\hline 1 & 1 & 2 & 3 \\
\hline 2 & 2 & 1 & 3 \\
\hline 3 & 3 & 3 & 3 \\
\hline
\end{tabular}

Consider a complex neutrosophic set $\mathcal{C}$ on $\mathcal{S}$ as:

$$
\mathcal{C}=\left\{\begin{array}{c}
\left\langle 1,0.9 e^{0.7 \pi i}, 0.7 e^{0.6 \pi i}, 0.5 e^{0.4 \pi i}\right\rangle, \\
\left\langle 2,0.8 e^{0.6 \pi i}, 0.6 e^{0.5 \pi i}, 0.4 e^{0.3 \pi i}\right\rangle, \\
\left\langle 3,0.5 e^{0.4 \pi i}, 0.4 e^{0.2 \pi i}, 0.3 e^{0.2 \pi i}\right\rangle
\end{array}\right\}
$$

Then clearly $\mathcal{C}$ is a complex neutrosophic subsemigroup of $\mathcal{S}$.

\subsection{Cartesian Product of Complex Neutrosophic Subsemigroups.}

Definition 3.2. Let

$$
\mathcal{C}_{1}=\left\{\left\langle\mathcal{C}_{1 T}=p_{\mathcal{C}_{1}} e^{i \mu_{\mathcal{C}_{1}}}, \mathcal{C}_{1 I}=q_{\mathcal{C}_{1}} e^{i \nu_{\mathcal{C}_{1}}}, \mathcal{C}_{1 F}=r_{\mathcal{C}_{1}} e^{i \omega_{\mathcal{C}_{1}}}\right\rangle\right\}
$$

and

$$
\mathcal{C}_{2}=\left\{\left\langle\mathcal{C}_{2 T}=p_{\mathcal{C}_{2}} e^{i \mu_{\mathcal{C}_{2}}}, \mathcal{C}_{2 I}=q_{\mathcal{C}_{2}} e^{i \nu_{\mathcal{C}_{2}}}, \mathcal{C}_{2 F}=r_{\mathcal{C}_{2}} e^{i \omega_{\mathcal{C}_{2}}}\right\rangle\right\}
$$


be any two complex neutrosophic subsemigroups of the semigroups $\mathcal{S}_{1}$ and $\mathcal{S}_{2}$ respectively. Then the Cartesian product of $\mathcal{C}_{1}$ and $\mathcal{C}_{2}$ denoted by $\mathcal{C}_{1} \times \mathcal{C}_{2}$ is defined as:

$$
\mathcal{C}_{1} \times \mathcal{C}_{2}=\left\{\left\langle\begin{array}{c}
(x, y),\left(\mathcal{C}_{1} \times \mathcal{C}_{2}\right)_{T}(x, y),\left(\mathcal{C}_{1} \times \mathcal{C}_{2}\right)_{I}(x, y), \\
\left(\mathcal{C}_{1} \times \mathcal{C}_{2}\right)_{F}(x, y)
\end{array}\right) / \forall x \in \mathcal{S}_{1}, y \in \mathcal{S}_{2}\right\}
$$

where

$$
\begin{aligned}
\left(\mathcal{C}_{1} \times \mathcal{C}_{2}\right)_{T}(x, y) & =\min \left\{\mathcal{C}_{1 T}(x), \mathcal{C}_{2 T}(y)\right\} \\
\left(\mathcal{C}_{1} \times \mathcal{C}_{2}\right)_{I}(x, y) & =\min \left\{\mathcal{C}_{1 I}(x), \mathcal{C}_{2 I}(y)\right\} \\
\left(\mathcal{C}_{1} \times \mathcal{C}_{2}\right)_{F}(x, y) & =\max \left\{\mathcal{C}_{1 F}(x), \mathcal{C}_{2 F}(y)\right\}
\end{aligned}
$$

for all $x$ in $\mathcal{S}_{1}$ and $y$ in $\mathcal{S}_{2}$.

Example 3.2. Let $\mathcal{S}_{1}=\{1,2,3\}$ and $\mathcal{S}_{2}=\{a, b, c\}$ are any two semigroups with the following multiplication tables:

\begin{tabular}{|l|l|l|l|}
\hline$\cdot$ & 1 & 2 & 3 \\
\hline 1 & 1 & 2 & 3 \\
\hline 2 & 2 & 1 & 3 \\
\hline 3 & 3 & 3 & 3 \\
\hline
\end{tabular}

\begin{tabular}{|l|l|l|l|}
\hline$\cdot$ & $a$ & $b$ & $c$ \\
\hline$a$ & $c$ & $b$ & $a$ \\
\hline$b$ & $b$ & $b$ & $c$ \\
\hline$c$ & $c$ & $c$ & $c$ \\
\hline
\end{tabular}

Consider

$$
\mathcal{C}_{1}=\left\{\begin{array}{c}
\left\langle 1,0.9 e^{0.7 \pi i}, 0.7 e^{0.6 \pi i}, 0.5 e^{0.4 \pi i}\right\rangle,\left\langle 2,0.8 e^{0.6 \pi i}, 0.6 e^{0.5 \pi i}, 0.4 e^{0.3 \pi i}\right\rangle, \\
\left\langle 3,0.5 e^{0.4 \pi i}, 0.4 e^{0.2 \pi i}, 0.3 e^{0.2 \pi i}\right\rangle
\end{array}\right\}
$$

and

$$
\mathcal{C}_{2}=\left\{\begin{array}{c}
\left\langle a, 0.8 e^{0.7 \pi i}, 0.5 e^{0.3 \pi i}, 0.4 e^{0.4 \pi i}\right\rangle,\left\langle b, 0.6 e^{0.5 \pi i}, 0.5 e^{0.4 \pi i}, 0.3 e^{0.2 \pi i}\right\rangle, \\
\left\langle c, 0.8 e^{0.7 \pi i}, 0.7 e^{0.5 \pi i}, 0.3 e^{0.2 \pi i}\right\rangle
\end{array}\right\}
$$

be any two complex neutrosophic subsemigroups of $\mathcal{S}_{1}$ and $\mathcal{S}_{2}$, respectively. Now let $x=1$ and $y=a$, then

$$
\begin{aligned}
\mathcal{C}_{1} \times \mathcal{C}_{2}= & \left\{\left\langle\left(\mathcal{C}_{1} \times \mathcal{C}_{2}\right)_{T}(1, a),\left(\mathcal{C}_{1} \times \mathcal{C}_{2}\right)_{I}(1, a),\left(\mathcal{C}_{1} \times \mathcal{C}_{2}\right)_{F}(1, a)\right\rangle, \ldots\right\} \\
= & \left\{\left\langle\min \left\{\mathcal{C}_{1 T}(1), \mathcal{C}_{2 T}(a)\right\}, \min \left\{\mathcal{C}_{1 I}(1), \mathcal{C}_{2 I}(a)\right\}, \max \left\{\mathcal{C}_{1 F}(1),\right.\right.\right. \\
& \left.\left.\left.\mathcal{C}_{2 F}(a)\right\}\right\rangle, \ldots\right\} \\
= & \left\{\left\langle\min \left\{0.9 e^{0.7 \pi i}, 0.8 e^{0.7 \pi i}\right\}, \min \left\{0.7 e^{0.6 \pi i}, 0.5 e^{0.3 \pi i}\right\}, \max \left\{0.5 e^{0.4 \pi i},\right.\right.\right. \\
& \left.\left.\left.0.4 e^{0.4 \pi i}\right\}\right\rangle, \ldots\right\} \\
= & \left\{\left\langle 0.8 e^{0.7 \pi i}, 0.5 e^{0.3 \pi i}, 0.5 e^{0.4 \pi i}\right\rangle, \ldots\right\} .
\end{aligned}
$$

\section{Complex Neutrosophic Ideals}

In this section, we define some ideals namely complex neutrosophic (left, right, interior) ideal in semigroup, with the help of examples and study some of its related results. 


\subsection{Complex Neutrosophic Left Ideal.}

Definition 4.1. A complex neutrosophic set $\mathcal{C}=\left\{\left\langle T_{\mathcal{C}}=p_{\mathcal{C}} \cdot e^{i \mu_{\mathcal{C}}}, I_{\mathcal{C}}=q_{\mathcal{C}} \cdot e^{i \nu_{\mathcal{C}}}, F_{\mathcal{C}}=r_{\mathcal{C}} \cdot e^{i \omega_{\mathcal{C}}}\right\rangle\right\}$ on a semigroup $\mathcal{S}$ is known as a complex neutrosophic left ideal of $\mathcal{S}$, if

$$
\begin{aligned}
& \mathcal{C}(x y) \geq \mathcal{C}(y) \text { i.e., } \\
& \left(\text { i) } p_{\mathcal{C}}(x y) \cdot e^{i \mu_{\mathcal{C}}(x y)} \geq p_{\mathcal{C}}(y) \cdot e^{i \mu_{\mathcal{C}}(y)}\right. \\
& \text { (ii) } q_{\mathcal{C}}(x y) \cdot e^{i \nu_{\mathcal{C}}(x y)} \geq q_{\mathcal{C}}(y) \cdot e^{i \nu_{\mathcal{C}}(y)} \\
& \text { (iii) } r_{\mathcal{C}}(x y) \cdot e^{i \omega_{\mathcal{C}}(x y)} \leq r_{\mathcal{C}}(y) \cdot e^{i \omega_{\mathcal{C}}(y)}, \forall x, y \in \mathcal{S} .
\end{aligned}
$$

Example 4.1. Let $\mathcal{S}=\{a, b, c, d\}$ be a semigroup with the following multiplication table:

\begin{tabular}{|l|l|l|l|l|}
\hline$\cdot$ & $a$ & $b$ & $c$ & $d$ \\
\hline$a$ & $a$ & $a$ & $a$ & $a$ \\
\hline$b$ & $a$ & $a$ & $a$ & $a$ \\
\hline$c$ & $a$ & $a$ & $b$ & $a$ \\
\hline$d$ & $a$ & $a$ & $b$ & $b$ \\
\hline
\end{tabular}

Consider a complex neutrosophic set $\mathcal{C}$ on $\mathcal{S}$ as:

$$
\mathcal{C}=\left\{\begin{array}{c}
\left\langle a, 0.9 e^{0.6 \pi i}, 0.8 e^{0.5 \pi i}, 0.4 e^{0.3 \pi i}\right\rangle,\left\langle b, 0.7 e^{0.5 \pi i}, 0.6 e^{0.4 \pi i}, 0.5 e^{0.4 \pi i}\right\rangle, \\
\left\langle c, 0.6 e^{0.4 \pi i}, 0.4 e^{0.3 \pi i}, 0.7 e^{0.5 \pi i}\right\rangle,\left\langle d, 0.5 e^{0.5 \pi i}, 0.4 e^{0.3 \pi i}, 0.7 e^{0.5 \pi i}\right\rangle
\end{array}\right\}
$$

Then $\mathcal{C}$ is a complex neutrosophic left ideal of $\mathcal{S}$.

\subsection{Complex Neutrosophic Right Ideal.}

Definition 4.2. A complex neutrosophic set $\mathcal{C}=\left\{\left\langle T_{\mathcal{C}}=p_{\mathcal{C}} \cdot e^{i \mu_{\mathcal{C}}}, I_{\mathcal{C}}=q_{\mathcal{C}} \cdot e^{i \nu_{\mathcal{C}}}, F_{\mathcal{C}}=r_{\mathcal{C}} \cdot e^{i \omega_{\mathcal{C}}}\right\rangle\right\}$ on a semigroup $\mathcal{S}$ is known as a complex neutrosophic right ideal of $\mathcal{S}$, if

$\mathcal{C}(x y) \geq \mathcal{C}(x)$ i.e.,

(i) $p_{\mathcal{C}}(x y) \cdot e^{i \mu_{\mathcal{C}}(x y)} \geq p_{\mathcal{C}}(x) \cdot e^{i \mu_{\mathcal{C}}(x)}$

(ii) $q_{\mathcal{C}}(x y) \cdot e^{i \nu_{\mathcal{C}}(x y)} \geq q_{\mathcal{C}}(x) \cdot e^{i \nu_{\mathcal{C}}(x)}$

(iii) $r_{\mathcal{C}}(x y) \cdot e^{i \omega_{\mathcal{C}}(x y)} \leq r_{\mathcal{C}}(x) \cdot e^{i \omega_{\mathcal{C}}(x)}, \forall x, y \in \mathcal{S}$.

\subsection{Complex Neutrosophic Ideal.}

Definition 4.3. A complex neutrosophic set $\mathcal{C}=\left\{\left\langle T_{\mathcal{C}}=p_{\mathcal{C}} \cdot e^{i \mu_{\mathcal{C}}}, I_{\mathcal{C}}=q_{\mathcal{C}} \cdot e^{i \nu_{\mathcal{C}}}, F_{\mathcal{C}}=r_{\mathcal{C}} \cdot e^{i \omega_{\mathcal{C}}}\right\rangle\right\}$ on a semigroup $\mathcal{S}$ is known as a complex neutrosophic ideal of $\mathcal{S}$, if it is both a complex neutrosophic left ideal and a complex neutrosophic right ideal of $\mathcal{S}$. 
Example 4.2. Let $\mathcal{S}=\{a, b, c\}$ be a semigroup with the following Cayley table:

\begin{tabular}{|l|l|l|l|}
\hline$\cdot$ & $a$ & $b$ & $c$ \\
\hline$a$ & $a$ & $a$ & $a$ \\
\hline$b$ & $a$ & $a$ & $a$ \\
\hline$c$ & $a$ & $a$ & $c$ \\
\hline
\end{tabular}

If we define a complex neutrosophic set $\mathcal{C}$ on $\mathcal{S}$ as:

$$
\mathcal{C}=\left\{\begin{array}{c}
\left\langle a, 0.8 e^{0.6 \pi i}, 0.6 e^{0.5 \pi i}, 0.5 e^{0.4 \pi i}\right\rangle,\left\langle b, 0.7 e^{0.6 \pi i}, 0.5 e^{0.4 \pi i}, 0.6 e^{0.4 \pi i}\right\rangle, \\
\left\langle c, 0.7 e^{0.5 \pi i}, 0.4 e^{0.3 \pi i}, 0.7 e^{0.5 \pi i}\right\rangle
\end{array}\right\}
$$

Then obviously $\mathcal{C}$ is a complex neutrosophic ideal of $\mathcal{S}$.

Remark 4.1. Every complex neutrosophic left (resp., right) ideal is a complex neutrosophic subsemigroup. But the converse may not be true as seen in the following example.

Example 4.3. Let $\mathcal{S}=\{a, b, c, d\}$ be a semigroup with the following Cayley table:

\begin{tabular}{|l|l|l|l|l|}
\hline$\cdot$ & $a$ & $b$ & $c$ & $d$ \\
\hline$a$ & $a$ & $a$ & $a$ & $a$ \\
\hline$b$ & $a$ & $a$ & $a$ & $a$ \\
\hline$c$ & $a$ & $a$ & $a$ & $b$ \\
\hline$d$ & $a$ & $a$ & $b$ & $c$ \\
\hline
\end{tabular}

Take a complex neutrosophic set $\mathcal{C}$ on $\mathcal{S}$ as:

$$
\mathcal{C}=\left\{\begin{array}{c}
\left\langle a, 0.8 e^{0.6 \pi i}, 0.6 e^{0.5 \pi i}, 0.5 e^{0.4 \pi i}\right\rangle,\left\langle b, 0.6 e^{0.6 \pi i}, 0.5 e^{0.4 \pi i}, 0.6 e^{0.4 \pi i}\right\rangle, \\
\left\langle c, 0.8 e^{0.5 \pi i}, 0.4 e^{0.3 \pi i}, 0.7 e^{0.5 \pi i}\right\rangle,\left\langle d, 0.4 e^{0.4 \pi i}, 0.3 e^{0.3 \pi i}, 0.7 e^{0.5 \pi i}\right\rangle
\end{array}\right\}
$$

Then clearly $\mathcal{C}$ is a complex neutrosophic subsemigroup of $\mathcal{S}$. However it is not a complex neutrosophic right ideal of $\mathcal{S}$, because

$$
T_{\mathcal{C}}(c d)=T_{\mathcal{C}}(b)=0.6 e^{0.6 \pi i} \ngtr 0.8 e^{0.5 \pi i}=T_{\mathcal{C}}(c) .
$$

\subsection{Complex Neutrosophic Interior Ideal.}

Definition 4.4. A complex neutrosophic set $\mathcal{C}=\left\{\left\langle T_{\mathcal{C}}=p_{\mathcal{C}} \cdot e^{i \mu_{\mathcal{C}}}, I_{\mathcal{C}}=q_{\mathcal{C}} \cdot e^{i \nu_{\mathcal{C}}}, F_{\mathcal{C}}=r_{\mathcal{C}} \cdot e^{i \omega_{\mathcal{C}}}\right\rangle\right\}$ on a semigroup $\mathcal{S}$ is known as a complex neutrosophic interior ideal of $\mathcal{S}$, if

$$
\begin{aligned}
& \mathcal{C}(x \kappa y) \geq \mathcal{C}(\kappa) \text { i.e., } \\
& (i) p_{\mathcal{C}}(x \kappa y) \cdot e^{i \mu_{\mathcal{C}}(x \kappa y)} \geq p_{\mathcal{C}}(\kappa) \cdot e^{i \mu_{\mathcal{C}}(\kappa)} \\
& (i i) q_{\mathcal{C}}(x \kappa y) \cdot e^{i \nu_{\mathcal{C}}(x \kappa y)} \geq q_{\mathcal{C}}(\kappa) \cdot e^{i \nu_{\mathcal{C}}(\kappa)} \\
& (i i i) r_{\mathcal{C}}(x \kappa y) \cdot e^{i \omega_{\mathcal{C}}(x \kappa y)} \leq r_{\mathcal{C}}(\kappa) \cdot e^{i \omega_{\mathcal{C}}(\kappa)}, \forall x, \kappa, y \in \mathcal{S} .
\end{aligned}
$$


Example 4.4. Let $\mathcal{S}=\{a, b, c, d\}$ be a semigroup with the following multiplication table:

\begin{tabular}{|l|l|l|l|l|}
\hline$\cdot$ & $a$ & $b$ & $c$ & $d$ \\
\hline$a$ & $a$ & $a$ & $a$ & $a$ \\
\hline$b$ & $a$ & $a$ & $a$ & $a$ \\
\hline$c$ & $a$ & $a$ & $b$ & $a$ \\
\hline$d$ & $a$ & $a$ & $b$ & $b$ \\
\hline
\end{tabular}

Consider a complex neutrosophic set $\mathcal{C}$ on $\mathcal{S}$ as:

$$
\mathcal{C}=\left\{\begin{array}{c}
\left\langle a, 0.7 e^{0.6 \pi i}, 0.6 e^{0.4 \pi i}, 0.3 e^{0.5 \pi i}\right\rangle,\left\langle b, 0,0.5 e^{0.4 \pi i}, 0.5 e^{0.6 \pi i}\right\rangle, \\
\left\langle c, 0.5 e^{0.4 \pi i}, 0.4 e^{0.3 \pi i}, 0.7 e^{0.7 \pi i}\right\rangle,\left\langle d, 0,0.3 e^{0.2 \pi i}, 0.7 e^{0.7 \pi i}\right\rangle
\end{array}\right\}
$$

Then $\mathcal{C}$ is a complex neutrosophic interior ideal of $\mathcal{S}$.

Remark 4.2. Every complex neutrosophic ideal is a complex neutrosophic interior ideal. But the converse may not be true as seen in the Example 4.4. For

left

$$
T_{\mathcal{C}}(d c)=T_{\mathcal{C}}(b)=0 \nsupseteq 0.5 e^{0.4 \pi i}=T_{\mathcal{C}}(c)
$$

right

$$
T_{\mathcal{C}}(d c)=T_{\mathcal{C}}(b)=0 \geq 0=T_{\mathcal{C}}(d) .
$$

So it is a complex neutrosophic right ideal but not a left ideal. Hence $\mathcal{C}$ is not a complex neutrosophic ideal.

\section{Characteristic Function of Complex Neutrosophic Set}

Definition 5.1. Let $H$ be a non-empty subset over the universe $\mathcal{X}$. Then the characteristic complex neutrosophic function of $H$ in $\mathcal{X}$, defined to be a structure:

$$
C_{H}=\left\{\left\langle x, T_{C_{H}}(x), I_{C_{H}}(x), F_{C_{H}}(x)\right\rangle: x \in H\right\}
$$

where

$$
\begin{aligned}
& T_{C_{H}}(x)= \begin{cases}1 \cdot e^{i 2 \pi} & \text { if } x \in H \\
0 & \text { otherwise }\end{cases} \\
& I_{C_{H}}(x)= \begin{cases}1 \cdot e^{i 2 \pi} & \text { if } x \in H \\
0 & \text { otherwise }\end{cases} \\
& F_{C_{H}}(x)= \begin{cases}0 & \text { if } x \in H \\
1 \cdot e^{i 2 \pi} & \text { otherwise }\end{cases}
\end{aligned}
$$

Definition 5.2. The characteristic function of whole complex neutrosophic set $\mathcal{S}$ in semigroup $\mathcal{S}$ is defined as;

$$
C_{\mathcal{S}}=\left\{\left\langle\left(\hat{1}_{T_{C_{\mathcal{S}}}}, 1 \cdot e^{i 2 \pi}\right),\left(\hat{1}_{I_{C_{\mathcal{S}}}}, 1 \cdot e^{i 2 \pi}\right),\left(\hat{0}_{F_{C_{\mathcal{S}}}}, 0\right)\right\rangle: x \in \mathcal{S}\right\}
$$




\subsection{Direct Product of Two Complex Neutrosophic Sets.}

Definition 5.3. Let

$$
\mathcal{C}_{1}=\left\langle\mathcal{C}_{1 T}=p_{\mathcal{C}_{1}} e^{i \mu_{\mathcal{C}_{1}}}, \mathcal{C}_{1 I}=q_{\mathcal{C}_{1}} e^{i \nu_{\mathcal{C}_{1}}}, \mathcal{C}_{1 F}=r_{\mathcal{C}_{1}} e^{i \omega_{\mathcal{C}_{1}}}\right\rangle
$$

and

$$
\mathcal{C}_{2}=\left\langle\mathcal{C}_{2 T}=p_{\mathcal{C}_{2}} e^{i \mu_{\mathcal{C}_{2}}}, \mathcal{C}_{2 I}=q_{\mathcal{C}_{2}} e^{i \nu_{\mathcal{C}_{2}}}, \mathcal{C}_{2 F}=r_{\mathcal{C}_{2}} e^{i \omega_{\mathcal{C}_{2}}}\right\rangle
$$

be any two complex neutrosophic sets on $\mathcal{S}$, then the product is define as;

$$
\mathcal{C}_{1} \otimes \mathcal{C}_{2}=\left\{\begin{array}{c}
\left\langle x,\left(p_{\mathcal{C}_{1}} \circ p_{\mathcal{C}_{2}}\right)(x) \cdot e^{i\left(\mu_{\mathcal{C}_{1}} \circ \mu_{\mathcal{C}_{2}}\right)(x)},\left(q_{\mathcal{C}_{1}} \circ q_{\mathcal{C}_{2}}\right)(x) \cdot e^{i\left(\nu_{\mathcal{C}_{1}} \circ \nu_{\mathcal{C}_{2}}\right)(x)},\right. \\
\left.\left(r_{\mathcal{C}_{1}} \circ r_{\mathcal{C}_{2}}\right)(x) \cdot e^{i\left(\omega_{\mathcal{C}_{1}} \circ \omega_{\mathcal{C}_{2}}\right)(x)}\right\rangle: x \in \mathcal{S}
\end{array}\right\}
$$

where

$$
\begin{aligned}
& \left(p_{\mathcal{C}_{1}} \circ p_{\mathcal{C}_{2}}\right)(x) \cdot e^{i\left(\mu_{\mathcal{C}_{1}} \circ \mu_{\mathcal{C}_{2}}\right)(x)}=\left\{\begin{array}{rr}
\sup _{x=y \kappa}\left[\min \left\{p_{\mathcal{C}_{1}}(y) e^{i \mu_{\mathcal{C}_{1}}(y)}, p_{\mathcal{C}_{2}}(\kappa) e^{i \mu_{\mathcal{C}_{2}}(\kappa)}\right\}\right] \\
\text { if } x=y \kappa \text { for some } y, \kappa \in \mathcal{S} \\
0 & \text { otherwise }
\end{array}\right. \\
& \left(q_{\mathcal{C}_{1}} \circ q_{\mathcal{C}_{2}}\right)(x) \cdot e^{i\left(\nu_{\mathcal{C}_{1}} \circ \nu_{\mathcal{C}_{2}}\right)(x)}=\left\{\begin{array}{r}
\sup _{x=y \kappa}\left[\min \left\{q_{\mathcal{C}_{1}}(y) e^{i \nu_{\mathcal{C}_{1}}(y)}, q_{\mathcal{C}_{2}}(\kappa) e^{i \nu_{\mathcal{C}_{2}}(\kappa)}\right\}\right] \\
\text { if } x=y \kappa \text { for some } y, \kappa \in \mathcal{S} \\
0 \quad \text { otherwise }
\end{array}\right. \\
& \left(r_{\mathcal{C}_{1}} \circ r_{\mathcal{C}_{2}}\right)(x) \cdot e^{i\left(\omega_{\mathcal{C}_{1}} \circ \omega_{\mathcal{C}_{2}}\right)(x)}=\left\{\begin{array}{c}
\inf _{x=y \kappa}\left[\max \left\{r_{\mathcal{C}_{1}}(y) e^{i \omega_{\mathcal{C}_{1}}(y)}, r_{\mathcal{C}_{2}}(\kappa) e^{i \omega_{\mathcal{C}_{2}}(\kappa)}\right\}\right] \\
\text { if } x=y \kappa \text { for some } y, \kappa \in \mathcal{S} \\
1 \cdot e^{i 2 \pi} \quad \text { otherwise }
\end{array}\right.
\end{aligned}
$$

for all $x$ in $\mathcal{S}$.

Proposition 5.1. A complex neutrosophic sets $\mathcal{C}_{1}, \mathcal{C}_{2}$ and $\mathcal{C}_{3}$ of a semigroup $\mathcal{S}$, if $\mathcal{C}_{1} \subseteq \mathcal{C}_{2}$, then $\mathcal{C}_{1} \otimes \mathcal{C}_{3} \subseteq$ $\mathcal{C}_{2} \otimes \mathcal{C}_{3}$ and $\mathcal{C}_{3} \otimes \mathcal{C}_{1} \subseteq \mathcal{C}_{3} \otimes \mathcal{C}_{2}$

Proof: We are proving $\mathcal{C}_{1} \otimes \mathcal{C}_{3} \subseteq \mathcal{C}_{2} \otimes \mathcal{C}_{3}$.

Since $\mathcal{C}_{1}, \mathcal{C}_{2}$ and $\mathcal{C}_{3}$ are complex neutrosophic sets of $\mathcal{S}$. Let $x \in \mathcal{S}$.

Case 1: If $x$ is not expressed as $x=y \kappa$, then

$\left(\mathcal{C}_{1} \otimes \mathcal{C}_{3}\right)(x)=\langle\hat{0}, \hat{0}, \hat{1}\rangle$ and $\left(\mathcal{C}_{2} \otimes \mathcal{C}_{3}\right)(x)=\langle\hat{0}, \hat{0}, \hat{1}\rangle$.

Clearly, $\mathcal{C}_{1} \otimes \mathcal{C}_{3} \subseteq \mathcal{C}_{2} \otimes \mathcal{C}_{3}$.

Case 2: Assume that there exist $y, \kappa \in \mathcal{S}$, such that $x=y \kappa$. Then

$$
\begin{aligned}
\left(p_{\mathcal{C}_{1}} \circ p_{\mathcal{C}_{3}}\right)(x) \cdot e^{i\left(\mu_{\mathcal{C}_{1}} \circ \mu_{\mathcal{C}_{3}}\right)(x)} & =\sup _{x=y \kappa}\left[\min \left\{p_{\mathcal{C}_{1}}(y) e^{i \mu_{\mathcal{C}_{1}}(y)}, p_{\mathcal{C}_{3}}(\kappa) e^{i \mu_{\mathcal{C}_{3}}(\kappa)}\right\}\right] \\
& \leq \sup _{x=y \kappa}\left[\min \left\{p_{\mathcal{C}_{2}}(y) e^{i \mu_{\mathcal{C}_{2}}(y)}, p_{\mathcal{C}_{3}}(\kappa) e^{i \mu_{\mathcal{C}_{3}}(\kappa)}\right\}\right] \\
& =\left(p_{\mathcal{C}_{2}} \circ p_{\mathcal{C}_{3}}\right)(x) \cdot e^{i\left(\mu_{\mathcal{C}_{2}} \circ \mu_{\mathcal{C}_{3}}\right)(x)} .
\end{aligned}
$$


Similarly,

$$
\left(q_{\mathcal{C}_{1}} \circ q_{\mathcal{C}_{3}}\right)(x) \cdot e^{i\left(\nu_{\mathcal{C}_{1}} \circ \nu_{\mathcal{C}_{3}}\right)(x)} \leq\left(q_{\mathcal{C}_{2}} \circ q_{\mathcal{C}_{3}}\right)(x) \cdot e^{i\left(\nu_{\mathcal{C}_{2}} \circ \nu_{\mathcal{C}_{3}}\right)(x)}
$$

And

$$
\begin{aligned}
\left(r_{\mathcal{C}_{1}} \circ r_{\mathcal{C}_{3}}\right)(x) \cdot e^{i\left(\omega_{\mathcal{C}_{1}} \circ \omega_{\mathcal{C}_{3}}\right)(x)} & =\inf \left[\max \left\{r_{\mathcal{C}_{1}}(y) e^{i \omega_{\mathcal{C}_{1}}(y)}, r_{\mathcal{C}_{3}}(\kappa) e^{i \omega_{\mathcal{C}_{3}}(\kappa)}\right\}\right] \\
& \geq \inf \left[\max \left\{r_{\mathcal{C}_{2}}(y) e^{i \omega_{\mathcal{C}_{2}}(y)}, r_{\mathcal{C}_{3}}(\kappa) e^{i \omega_{\mathcal{C}_{3}}(\kappa)}\right\}\right] \\
& =\left(r_{\mathcal{C}_{2}} \circ r_{\mathcal{C}_{3}}\right)(x) \cdot e^{i\left(\omega_{\mathcal{C}_{2}} \circ \omega_{\mathcal{C}_{3}}\right)(x)} .
\end{aligned}
$$

Therefore, $\mathcal{C}_{1} \otimes \mathcal{C}_{3} \subseteq \mathcal{C}_{2} \otimes \mathcal{C}_{3}$. Similarly we can proved $\mathcal{C}_{3} \otimes \mathcal{C}_{1} \subseteq \mathcal{C}_{3} \otimes \mathcal{C}_{2}$.

Proposition 5.2. Let $H$ and $K$ be any subsets of a semigroup $\mathcal{S}$, we have

(1) $C_{H} \otimes C_{K}=C_{H K} \Rightarrow\left\langle T_{C_{H}} \circ T_{C_{K}}, I_{C_{H}} \circ I_{C_{K}}, F_{C_{H}} \circ F_{C_{K}}\right\rangle=\left\langle T_{C_{H K}}, I_{C_{H K}}, F_{C_{H K}}\right\rangle$.

(2) $C_{H} \cup C_{K}=C_{H \cup K} \Rightarrow\left\langle T_{C_{H}} \cup T_{C_{K}}, I_{C_{H}} \cup I_{C_{K}}, F_{C_{H}} \cap F_{C_{K}}\right\rangle=\left\langle T_{C_{H \cup K}}, I_{C_{H \cup K}}, F_{C_{H \cap K}}\right\rangle$.

(3) $C_{H} \cap C_{K}=C_{H \cap K} \Rightarrow\left\langle T_{C_{H}} \cap T_{C_{K}}, I_{C_{H}} \cap I_{C_{K}}, F_{C_{H}} \cup F_{C_{K}}\right\rangle=\left\langle T_{C_{H \cap K}}, I_{C_{H \cap K}}, F_{C_{H \cup K}}\right\rangle$.

Proof: (1) Let $\alpha \in \mathcal{S}$. If $\alpha \in H K$, then

$T_{C_{H K}}(\alpha)=1 . e^{i 2 \pi}, I_{C_{H K}}(\alpha)=1 . e^{i 2 \pi}$ and $F_{C_{H K}}(\alpha)=0$ and $\alpha=m n$ for some $m \in H$ and $n \in K$.

Thus,

$$
\begin{aligned}
\left(T_{C_{H}} \circ T_{C_{K}}\right)(\alpha) & =\sup _{\alpha=x y}\left\{\min \left\{T_{C_{H}}(x), T_{C_{K}}(y)\right\}\right\} \\
& \geq \min \left\{T_{C_{H}}(m), T_{C_{K}}(n)\right\}=1 . e^{i 2 \pi} \\
\left(I_{C_{H}} \circ I_{C_{K}}\right)(\alpha) & =\sup _{\alpha=x y}\left\{\min \left\{I_{C_{H}}(x), I_{C_{K}}(y)\right\}\right\} \\
& \geq \min \left\{I_{C_{H}}(m), I_{C_{K}}(n)\right\}=1 . e^{i 2 \pi}
\end{aligned}
$$

and

$$
\begin{aligned}
\left(F_{C_{H}} \circ F_{C_{K}}\right)(\alpha) & =\inf _{\alpha=x y}\left\{\max \left\{F_{C_{H}}(x), F_{C_{K}}(y)\right\}\right\} \\
& \leq \max \left\{F_{C_{H}}(m), F_{C_{K}}(n)\right\}=0 .
\end{aligned}
$$

It follows that,

$\left(T_{C_{H}} \circ T_{C_{K}}\right)(\alpha)=1 . e^{i 2 \pi},\left(I_{C_{H}} \circ I_{C_{K}}\right)(\alpha)=1 . e^{i 2 \pi}$ and $\left(F_{C_{H}} \circ F_{C_{K}}\right)(\alpha)=0$.

Therefore,

$\left\langle T_{C_{H}} \circ T_{C_{K}}, I_{C_{H}} \circ I_{C_{K}}, F_{C_{H}} \circ F_{C_{K}}\right\rangle=\left\langle T_{C_{H K}}, I_{C_{H K}}, F_{C_{H K}}\right\rangle \Rightarrow C_{H} \otimes C_{K}=C_{H K}$.

Assume that $\alpha \notin H K$, then

$T_{C_{H K}}(\alpha)=0, I_{C_{H K}}(\alpha)=0$ and $F_{C_{H K}}(\alpha)=1 . e^{i 2 \pi}$.

Let $y, \kappa \in \mathcal{S}$ be such that $\alpha=y \kappa$, then we know that $y \notin H$ or $\kappa \notin K$.

Assume that $y \notin H$, then 


$$
\begin{aligned}
\left(T_{C_{H}} \circ T_{C_{K}}\right)(\alpha) & =\sup _{\alpha=y \kappa}\left\{\min \left\{T_{C_{H}}(y), T_{C_{K}}(\kappa)\right\}\right\} \\
& =\sup _{\alpha=y \kappa}\left\{\min \left\{0, T_{C_{K}}(\kappa)\right\}\right\} \\
& =0=T_{C_{H}}(\alpha) \\
\left(I_{C_{H}} \circ I_{C_{K}}\right)(\alpha) & =\sup _{\alpha=y \kappa}\left\{\min \left\{I_{C_{H}}(y), I_{C_{K}}(\kappa)\right\}\right\} \\
& =\sup _{\alpha=y \kappa}\left\{\min \left\{0, I_{C_{K}}(\kappa)\right\}\right\} \\
& =0=I_{C_{H K}}(\alpha)
\end{aligned}
$$

and

$$
\begin{aligned}
\left(F_{C_{H}} \circ F_{C_{K}}\right)(\alpha) & =\inf _{\alpha=y \kappa}\left\{\max \left\{F_{C_{H}}(y), F_{C_{K}}(\kappa)\right\}\right\} \\
& =\inf _{\alpha=y \kappa}\left\{\max \left\{1 . e^{i 2 \pi}, F_{C_{K}}(\kappa)\right\}\right\} \\
& =1 . e^{i 2 \pi}=F_{C_{H}}(\alpha) .
\end{aligned}
$$

Similarly, if $\kappa \notin K$, then

$\left(T_{C_{H}} \circ T_{C_{K}}\right)(\alpha)=0=T_{C_{H K}}(\alpha),\left(I_{C_{H}} \circ I_{C_{K}}\right)(\alpha)=0=I_{C_{H K}}(\alpha)$ and $\left(F_{C_{H}} \circ F_{C_{K}}\right)(\alpha)=1 . e^{i 2 \pi}=$ $F_{C_{H K}}(\alpha)$.

Therefore $C_{H} \otimes C_{K}=C_{H K}$.

Proof of (2) and (3) are straightforward.

Theorem 5.1. A complex neutrosophic set $\mathcal{C}$ on a semigroup $\mathcal{S}$ is a complex neutrosophic subsemigroup of $\mathcal{S}$ if and only if $\mathcal{C} \otimes \mathcal{C} \subseteq \mathcal{C}$.

Proof: Let $\mathcal{C}$ be a complex neutrosophic subsemigroup of $\mathcal{S}$, and $x \in \mathcal{S}$.

Case 1: If $x \neq y \kappa$, for any $y, \kappa \in \mathcal{S}$, then obviously $\mathcal{C} \otimes \mathcal{C} \subseteq \mathcal{C}$.

Case 2: If $x=y \kappa$, for any $y, \kappa \in \mathcal{S}$, then

$$
\begin{aligned}
\left(p_{\mathcal{C}} \circ p_{\mathcal{C}}\right)(x) \cdot e^{i\left(\mu_{\mathcal{C}} \circ \mu_{\mathcal{C}}\right)(x)} & =\sup _{x=y \kappa}\left[\min \left\{p_{\mathcal{C}}(y) e^{i \mu_{\mathcal{C}}(y)}, p_{\mathcal{C}}(\kappa) e^{i \mu_{\mathcal{C}}(\kappa)}\right\}\right] \\
& \leq \sup _{x=y \kappa}\left[p_{\mathcal{C}}(y \kappa) e^{i \mu_{\mathcal{C}}(y \kappa)}\right] \\
& =p_{\mathcal{C}}(x) \cdot e^{i \mu_{\mathcal{C}}(x)}
\end{aligned}
$$

Similarly,

$$
\left(q_{\mathcal{C}} \circ q_{\mathcal{C}}\right)(x) \cdot e^{i\left(\nu_{\mathcal{C}} \circ \nu_{\mathcal{C}}\right)(x)} \leq q_{\mathcal{C}}(x) \cdot e^{i \nu_{\mathcal{C}}(x)}
$$


And

$$
\begin{aligned}
\left(r_{\mathcal{C}} \circ r_{\mathcal{C}}\right)(x) \cdot e^{i\left(\omega_{\mathcal{C}} \circ \omega_{\mathcal{C}}\right)(x)} & =\inf _{x=y \kappa}\left[\max \left\{r_{\mathcal{C}}(y) \cdot e^{i \omega_{\mathcal{C}}(y)}, r_{\mathcal{C}}(\kappa) \cdot e^{i \omega_{\mathcal{C}}(\kappa)}\right\}\right] \\
& \geq \inf _{x=y \kappa}\left[r_{\mathcal{C}}(y \kappa) \cdot e^{i \omega_{\mathcal{C}}(y \kappa)}\right] \\
& =r_{\mathcal{C}}(x) \cdot e^{i \omega_{\mathcal{C}}(x)}
\end{aligned}
$$

Therefore, $\mathcal{C} \otimes \mathcal{C} \subseteq \mathcal{C}$.

Conversely, Suppose $\mathcal{C} \otimes \mathcal{C} \subseteq \mathcal{C}$, and assume $x=y \kappa$, then

$$
\begin{aligned}
p_{\mathcal{C}}(y \kappa) \cdot e^{i \mu_{\mathcal{C}}(y \kappa)} & \geq\left(p_{\mathcal{C}} \circ p_{\mathcal{C}}\right)(y \kappa) \cdot e^{i\left(\mu_{\mathcal{C}} \circ \mu_{\mathcal{C}}\right)(y \kappa)} \\
& =\sup _{y \kappa=y \kappa}\left[\min \left\{p_{\mathcal{C}}(y) e^{i \mu_{\mathcal{C}}(y)}, p_{\mathcal{C}}(\kappa) e^{i \mu_{\mathcal{C}}(\kappa)}\right\}\right] \\
& =\min \left\{p_{\mathcal{C}}(y) e^{i \mu_{\mathcal{C}}(y)}, p_{\mathcal{C}}(\kappa) e^{i \mu_{\mathcal{C}}(\kappa)}\right\} .
\end{aligned}
$$

Similarly,

$$
q_{\mathcal{C}}(y \kappa) \cdot e^{i \nu_{\mathcal{C}}(y \kappa)} \geq \min \left\{q_{\mathcal{C}}(y) e^{i \nu_{\mathcal{C}}(y)}, q_{\mathcal{C}}(\kappa) e^{i \nu_{\mathcal{C}}(\kappa)}\right\} .
$$

And

$$
\begin{aligned}
r_{\mathcal{C}}(y \kappa) \cdot e^{i \omega_{\mathcal{C}}(y \kappa)} & \left.\leq\left(r_{\mathcal{C}} \circ r_{\mathcal{C}}\right)(y \kappa) \cdot e^{i\left(\omega_{\mathcal{C}} \circ \omega_{\mathcal{C}}\right)(y \kappa)}\right) \\
& =\inf _{y \kappa=y \kappa}\left[\max \left\{r_{\mathcal{C}}(y) e^{i \omega_{\mathcal{C}}(y)}, r_{\mathcal{C}}(\kappa) e^{i \omega_{\mathcal{C}}(\kappa)}\right\}\right] \\
& =\max \left\{r_{\mathcal{C}}(y) e^{i \omega_{\mathcal{C}}(y)}, r_{\mathcal{C}}(\kappa) e^{i \omega_{\mathcal{C}}(\kappa)}\right\} .
\end{aligned}
$$

Hence $\mathcal{C}$ is a complex neutrosophic subsemigroup of $\mathcal{S}$.

Proposition 5.3. A complex neutrosophic set $\mathcal{C}$ on a semigroup $\mathcal{S}$, the following are equivalent:

(1) $\mathcal{C}$ is a complex neutrosophic left ideal of $\mathcal{S}$.

(2) $\mathcal{S} \otimes \mathcal{C} \subseteq \mathcal{C}$.

Proof: $(1) \Rightarrow(2)$ : Assume that $\mathcal{C}$ is a complex neutrosophic left ideal of $\mathcal{S}$. Let $x \in \mathcal{S}$, such that $(\mathcal{S} \otimes \mathcal{C})(x)=\langle\hat{0}, \hat{0}, \hat{1}\rangle$, then it is clear $\mathcal{S} \otimes \mathcal{C} \subseteq \mathcal{C}$.

Whenever there exist any two elements $y, \kappa \in \mathcal{S}$, such that $x=y \kappa$.

Then

$$
\begin{aligned}
\left(\hat{1}_{\mathcal{S}_{T}} \circ p_{\mathcal{C}} \cdot e^{i \mu_{\mathcal{C}}}\right)(x) & =\sup _{x=y \kappa}\left[\min \left\{\hat{1}_{\mathcal{S}_{T}}(y), p_{\mathcal{C}}(\kappa) \cdot e^{i \mu_{\mathcal{C}}(\kappa)}\right\}\right] \\
& \leq \sup _{x=y \kappa}\left[\min \left\{1 \cdot e^{i 2 \pi}, p_{\mathcal{C}}(y \kappa) \cdot e^{i \mu_{\mathcal{C}}(y \kappa)}\right\}\right] \\
& =p_{\mathcal{C}}(x) \cdot e^{i \mu_{\mathcal{C}}(x)} .
\end{aligned}
$$

Similarly,

$$
\left(\hat{1}_{\mathcal{S}_{I}} \circ q_{\mathcal{C}} \cdot e^{i \nu_{\mathcal{C}}}\right)(x) \leq q_{\mathcal{C}}(x) \cdot e^{i \nu_{\mathcal{C}}(x)} .
$$


And

$$
\begin{aligned}
\left(\hat{0}_{\mathcal{S}_{F}} \circ r_{\mathcal{C}} \cdot e^{i \omega}\right)(x) & =\inf _{x=y \kappa}\left[\max \left\{\hat{0}_{\mathcal{S}_{F}}(y), r_{\mathcal{C}}(\kappa) \cdot e^{i \omega(\kappa)}\right\}\right] \\
& \geq \inf _{x=y \kappa}\left[\max \left\{0, r_{\mathcal{C}}(y \kappa) \cdot e^{i \omega(y \kappa)}\right\}\right] \\
& =r_{\mathcal{C}}(x) \cdot e^{i \omega(x)}
\end{aligned}
$$

Therefore, $\mathcal{S} \otimes \mathcal{C} \subseteq \mathcal{C}$.

Conversely, $(2) \Rightarrow(1)$ : Suppose that $\mathcal{S} \otimes \mathcal{C} \subseteq \mathcal{C}$. For any elements $y, \kappa$ of $\mathcal{S}$, let $x=y \kappa$. Then

$$
\begin{aligned}
p_{\mathcal{C}}(y \kappa) \cdot e^{i \mu_{\mathcal{C}}(y \kappa)} & =p_{\mathcal{C}}(x) \cdot e^{i \mu_{\mathcal{C}}(x)} \\
& \geq\left(\hat{1}_{\mathcal{S}_{T}} \circ p_{\mathcal{C}} \cdot e^{i \mu_{\mathcal{C}}}\right)(x) \\
& =\sup _{x=y \kappa}\left[\min \left\{\hat{1}_{\mathcal{S}_{T}}(y), p_{\mathcal{C}}(\kappa) \cdot e^{i \mu_{\mathcal{C}}(\kappa)}\right\}\right] \\
& =p_{\mathcal{C}}(\kappa) \cdot e^{i \mu_{\mathcal{C}}(\kappa)} .
\end{aligned}
$$

Similarly,

$$
q_{\mathcal{C}}(y \kappa) \cdot e^{i \nu_{\mathcal{C}}(y \kappa)} \geq q_{\mathcal{C}}(\kappa) \cdot e^{i \nu_{\mathcal{C}}(\kappa)}
$$

And

$$
\begin{aligned}
r_{\mathcal{C}}(y \kappa) \cdot e^{i \omega_{\mathcal{C}}(y \kappa)} & =r_{\mathcal{C}}(x) \cdot e^{i \omega_{\mathcal{C}}(x)} \\
& \leq\left(\hat{0}_{\mathcal{S}_{F}} \circ r_{\mathcal{C}} \cdot e^{i \omega_{\mathcal{C}}}\right)(x) \\
& =\inf _{x=y \kappa}\left[\max \left\{\hat{0}_{\mathcal{S}_{F}}(y), r_{\mathcal{C}}(\kappa) \cdot e^{i \omega_{\mathcal{C}}(\kappa)}\right\}\right] \\
& =r_{\mathcal{C}}(\kappa) \cdot e^{i \omega_{\mathcal{C}}(\kappa)}
\end{aligned}
$$

Hence $\mathcal{C}$ is a complex neutrosophic left ideal of $\mathcal{S}$.

Proposition 5.4. A complex neutrosophic set $\mathcal{C}$ on a semigroup $\mathcal{S}$, the following are equivalent:

(1) $\mathcal{C}$ is a complex neutrosophic right ideal of $\mathcal{S}$.

(2) $\mathcal{C} \otimes \mathcal{S} \subseteq \mathcal{C}$.

Proof: Proof is similar to the Proposition 5.3.

Theorem 5.2. If $\mathcal{C}$ is a complex neutrosophic set of a semigroup $\mathcal{S}$, then $\mathcal{S} \otimes \mathcal{C}($ resp., $\mathcal{C} \otimes \mathcal{S})$ is a complex neutrosophic left (resp. right) ideal of $\mathcal{S}$.

Proof: Since $\mathcal{S} \otimes(\mathcal{S} \otimes \mathcal{C})=(\mathcal{S} \otimes \mathcal{S}) \otimes \mathcal{C} \subseteq \mathcal{S} \otimes \mathcal{C}$, it follows from Proposition 5.3, that $\mathcal{S} \otimes \mathcal{C}$ is a complex neutrosophic left ideal of $\mathcal{S}$. Similarly $\mathcal{C} \otimes \mathcal{S}$ is a complex neutrosophic right ideal of $\mathcal{S}$.

Theorem 5.3. Let $S$ be a left zero subsemigroup of a semigroup $\mathcal{S}$. If $\mathcal{C}$ is a complex neutrosophic left ideal of $\mathcal{S}$, then $\mathcal{C}(x)=\mathcal{C}(y)$ for all $x, y \in S$. 
Proof: Let $x, y \in S$. Then $x y=x$ and $y x=y$. Thus

$$
\begin{aligned}
p_{\mathcal{C}}(x) \cdot e^{i \mu_{\mathcal{C}}(x)} & =p_{\mathcal{C}}(x y) \cdot e^{i \mu_{\mathcal{C}}(x y)} \geq p_{\mathcal{C}}(y) \cdot e^{i \mu_{\mathcal{C}}(y)} \\
& =p_{\mathcal{C}}(y x) \cdot e^{i \mu_{\mathcal{C}}(y x)} \geq p_{\mathcal{C}}(x) \cdot e^{i \mu_{\mathcal{C}}(x)}
\end{aligned}
$$

Similarly,

$$
q_{\mathcal{C}}(x) \cdot e^{i \nu_{\mathcal{C}}(x)}=q_{\mathcal{C}}(y) \cdot e^{i \nu_{\mathcal{C}}(y)}
$$

And

$$
\begin{aligned}
r_{\mathcal{C}}(x) \cdot e^{i \omega_{\mathcal{C}}(x)} & =r_{\mathcal{C}}(x y) \cdot e^{i \omega_{\mathcal{C}}(x y)} \leq r_{\mathcal{C}}(y) \cdot e^{i \omega_{\mathcal{C}}(y)} \\
& =r_{\mathcal{C}}(y x) \cdot e^{i \omega_{\mathcal{C}}(y x)} \leq r_{\mathcal{C}}(x) \cdot e^{i \omega_{\mathcal{C}}(x)}
\end{aligned}
$$

Therefore, $\mathcal{C}(x)=\mathcal{C}(y)$ for all $x, y \in S$.

Theorem 5.4. Let $S$ be a right zero subsemigroup of a semigroup $\mathcal{S}$. If $\mathcal{C}$ is a complex neutrosophic right ideal of $\mathcal{S}$, then $\mathcal{C}(x)=\mathcal{C}(y)$ for all $x, y \in S$.

Proof: Proof is similar to the Theorem 5.3.

Theorem 5.5. Let $\mathcal{C}$ is a complex neutrosophic left ideal of a semigroup $\mathcal{S}$. If the set of all idempotent elements of $\mathcal{S}$ form a left zero subsemigroup of $\mathcal{S}$, then $\mathcal{C}(x)=\mathcal{C}(y)$ for all idempotent elements $x$ and $y$ of $\mathcal{S}$.

Proof: $\operatorname{Let} \operatorname{Idm}(\mathcal{S})$ be the set of all idempotent elements of $\mathcal{S}$ and assume that $\operatorname{Idm}(\mathcal{S})$ is a left zero subsemigroup of $\mathcal{S}$. For any $x, y \in \operatorname{Idm}(\mathcal{S})$, we have $x y=x$ and $y x=y$. Thus

$$
\begin{aligned}
p_{\mathcal{C}}(x) \cdot e^{i \mu_{\mathcal{C}}(x)} & =p_{\mathcal{C}}(x y) \cdot e^{i \mu_{\mathcal{C}}(x y)} \geq p_{\mathcal{C}}(y) \cdot e^{i \mu_{\mathcal{C}}(y)} \\
& =p_{\mathcal{C}}(y x) \cdot e^{i \mu_{\mathcal{C}}(y x)} \geq p_{\mathcal{C}}(x) \cdot e^{i \mu_{\mathcal{C}}(x)} \\
& =p_{\mathcal{C}}(y) \cdot e^{i \mu_{\mathcal{C}}(y)} .
\end{aligned}
$$

Similarly,

$$
q_{\mathcal{C}}(x) \cdot e^{i \nu_{\mathcal{C}}(x)}=q_{\mathcal{C}}(y) \cdot e^{i \nu_{\mathcal{C}}(y)}
$$

And

$$
\begin{aligned}
r_{\mathcal{C}}(x) \cdot e^{i \omega_{\mathcal{C}}(x)} & =r_{\mathcal{C}}(x y) \cdot e^{i \omega_{\mathcal{C}}(x y)} \leq r_{\mathcal{C}}(y) \cdot e^{i \omega_{\mathcal{C}}(y)} \\
& =r_{\mathcal{C}}(y x) \cdot e^{i \omega_{\mathcal{C}}(y x)} \leq r_{\mathcal{C}}(x) \cdot e^{i \omega_{\mathcal{C}}(x)} \\
& =r_{\mathcal{C}}(y) \cdot e^{i \omega_{\mathcal{C}}(y)} .
\end{aligned}
$$

Therefore, $\mathcal{C}(x)=\mathcal{C}(y)$ for all $x, y \in \operatorname{Idm}(\mathcal{S})$. 
Theorem 5.6. Let $\mathcal{C}$ is a complex neutrosophic right ideal of a semigroup $\mathcal{S}$. If the set of all idempotent elements of $\mathcal{S}$ form a right zero subsemigroup of $\mathcal{S}$, then $\mathcal{C}(x)=\mathcal{C}(y)$ for all idempotent elements $x$ and $y$ of $\mathcal{S}$.

Proof: Proof is similar to the Theorem 5.5.

Proposition 5.5. If $\mathcal{S}$ be a semigroup. Then the following properties are hold.

(1) The intersection of two complex neutrosophic subsemigroups of $\mathcal{S}$ is a complex neutrosophic subsemigroup of $\mathcal{S}$.

(2) The intersection of two complex neutrosophic left (resp., right) ideals of $\mathcal{S}$ is a complex neutrosophic left (resp., right) ideal of $\mathcal{S}$.

Proof: Let

$$
\mathcal{C}_{1}=\left\langle\mathcal{C}_{1 T}=p_{\mathcal{C}_{1}} \cdot e^{i \mu_{\mathcal{C}_{1}}}, \mathcal{C}_{1 I}=q_{\mathcal{C}_{1}} \cdot e^{i \nu_{\mathcal{C}_{1}}}, \mathcal{C}_{1 F}=r_{\mathcal{C}_{1}} \cdot e^{i \omega_{\mathcal{C}_{1}}}\right\rangle
$$

and

$$
\mathcal{C}_{2}=\left\langle\mathcal{C}_{2 T}=p_{\mathcal{C}_{2}} \cdot e^{i \mu_{\mathcal{C}_{2}}}, \mathcal{C}_{2 I}=q_{\mathcal{C}_{2}} \cdot e^{i \nu_{\mathcal{C}_{2}}}, \mathcal{C}_{2 F}=r_{\mathcal{C}_{2}} \cdot e^{i \omega_{\mathcal{C}_{2}}}\right\rangle
$$

be any two complex neutrosophic subsemigroups of $\mathcal{S}$. Let $x, y \in \mathcal{S}$. Then

$$
\begin{aligned}
& \left(p_{\mathcal{C}_{1}} \cdot e^{i \mu_{\mathcal{C}_{1}}} \cap p_{\mathcal{C}_{2}} \cdot e^{i \mu_{\mathcal{C}_{2}}}\right)(x y)=\min \left\{p_{\mathcal{C}_{1}}(x y) \cdot e^{i \mu_{\mathcal{C}_{1}}(x y)}, p_{\mathcal{C}_{2}}(x y) \cdot e^{i \mu_{\mathcal{C}_{2}}(x y)}\right\} \\
& \geq \min \left\{\min \left\{p_{\mathcal{C}_{1}}(x) \cdot e^{i \mu_{\mathcal{C}_{1}}(x)}, p_{\mathcal{C}_{1}}(y) \cdot e^{i \mu_{\mathcal{C}_{1}}(y)}\right\},\right. \\
& \left.\min \left\{p_{\mathcal{C}_{2}}(x) \cdot e^{i \mu_{\mathcal{C}_{2}}(x)}, p_{\mathcal{C}_{2}}(y) \cdot e^{i \mu_{\mathcal{C}_{2}}(y)}\right\}\right\} \\
& =\min \left\{\min \left\{p_{\mathcal{C}_{1}}(x) \cdot e^{i \mu_{\mathcal{C}_{1}}(x)}, p_{\mathcal{C}_{2}}(x) \cdot e^{i \mu_{\mathcal{C}_{2}}(x)}\right\},\right. \\
& \left.\min \left\{p_{\mathcal{C}_{1}}(y) \cdot e^{i \mu_{\mathcal{C}_{1}}(y)}, p_{\mathcal{C}_{2}}(y) \cdot e^{i \mu_{\mathcal{C}_{2}}(y)}\right\}\right\} \\
& =\min \left\{\left(p_{\mathcal{C}_{1}} \cdot e^{i \mu_{\mathcal{C}_{1}}} \cap p_{\mathcal{C}_{2}} \cdot e^{i \mu_{\mathcal{C}_{2}}}\right)(x),\right. \\
& \left.\left(p_{\mathcal{C}_{1}} \cdot e^{i \mu_{\mathcal{C}_{1}}} \cap p_{\mathcal{C}_{2}} \cdot e^{i \mu_{\mathcal{C}_{2}}}\right)(y)\right\} .
\end{aligned}
$$

Similarly,

$$
\begin{aligned}
\left(q_{\mathcal{C}_{1}} \cdot e^{i \nu_{\mathcal{C}_{1}}} \cap q_{\mathcal{C}_{2}} \cdot e^{i \nu_{\mathcal{C}_{2}}}\right)(x y) \geq & \min \left\{\left(q_{\mathcal{C}_{1}} \cdot e^{i \nu_{\mathcal{C}_{1}}} \cap q_{\mathcal{C}_{2}} \cdot e^{i \nu_{\mathcal{C}_{2}}}\right)(x),\right. \\
& \left.\left(q_{\mathcal{C}_{1}} \cdot e^{i \nu_{\mathcal{C}_{1}}} \cap q_{\mathcal{C}_{2}} \cdot e^{i \nu_{\mathcal{C}_{2}}}\right)(y)\right\}
\end{aligned}
$$


And

$$
\begin{aligned}
& \left(r_{\mathcal{C}_{1}} \cdot e^{i \omega_{\mathcal{C}_{1}}} \cup r_{\mathcal{C}_{2}} \cdot e^{i \omega_{\mathcal{C}_{2}}}\right)(x y)=\max \left\{r_{\mathcal{C}_{1}}(x y) \cdot e^{i \omega_{\mathcal{C}_{1}}(x y)}, r_{\mathcal{C}_{2}}(x y) \cdot e^{i \omega_{\mathcal{C}_{2}}(x y)}\right\} \\
& \leq \max \left\{\max \left\{r_{\mathcal{C}_{1}}(x) \cdot e^{i \omega_{\mathcal{C}_{1}}(x)}, r_{\mathcal{C}_{1}}(y) \cdot e^{i \omega_{\mathcal{C}_{1}}(y)}\right\}\right. \\
& \left.\max \left\{r_{\mathcal{C}_{2}}(x) \cdot e^{i \omega_{\mathcal{C}_{2}}(x)}, r_{\mathcal{C}_{2}}(y) \cdot e^{i \omega_{\mathcal{C}_{2}}(y)}\right\}\right\} \\
& =\max \left\{\max \left\{r_{\mathcal{C}_{1}}(x) \cdot e^{i \omega_{\mathcal{C}_{1}}(x)}, r_{\mathcal{C}_{2}}(x) \cdot e^{i \omega_{\mathcal{C}_{2}}(x)}\right\},\right. \\
& \left.\max \left\{r_{\mathcal{C}_{1}}(y) \cdot e^{i \omega_{\mathcal{C}_{1}}(y)}, r_{\mathcal{C}_{2}}(y) \cdot e^{i \omega_{\mathcal{C}_{2}}(y)}\right\}\right\} \\
& =\max \left\{\left(r_{\mathcal{C}_{1}} \cdot e^{i \omega_{\mathcal{C}_{1}}} \cup r_{\mathcal{C}_{2}} \cdot e^{i \omega_{\mathcal{C}_{2}}}\right)(x),\right. \\
& \left.\left(r_{\mathcal{C}_{1}} \cdot e^{i \omega_{\mathcal{C}_{1}}} \cup r_{\mathcal{C}_{2}} \cdot e^{i \omega_{\mathcal{C}_{2}}}\right)(y)\right\}
\end{aligned}
$$

Therefore, $\mathcal{C}_{1} \cap \mathcal{C}_{2}$ is a complex neutrosophic subsemigroup of $\mathcal{S}$.

(2) Let $\mathcal{C}_{1}$ and $\mathcal{C}_{2}$ be any two complex neutrosophic left ideals of semigroup $\mathcal{S}$, and $x, y \in \mathcal{S}$. Then

$$
\begin{aligned}
\left(p_{\mathcal{C}_{1}} \cdot e^{\left.i \mu_{\mathcal{C}_{1}} \cap p_{\mathcal{C}_{2}} \cdot e^{i \mu_{\mathcal{C}_{2}}}\right)(x y)}\right. & =\min \left\{p_{\mathcal{C}_{1}}(x y) \cdot e^{i \mu_{\mathcal{C}_{1}}(x y)}, p_{\mathcal{C}_{2}}(x y) \cdot e^{i \mu_{\mathcal{C}_{2}}(x y)}\right\} \\
& \geq \min \left\{p_{\mathcal{C}_{1}}(y) \cdot e^{i \mu_{\mathcal{C}_{1}}(y)}, p_{\mathcal{C}_{2}}(y) \cdot e^{i \mu_{\mathcal{C}_{2}}(y)}\right\} \\
& =\left(p_{\mathcal{C}_{1}} \cdot e^{i \mu_{\mathcal{C}_{1}}} \cap p_{\mathcal{C}_{2}} \cdot e^{i \mu_{\mathcal{C}_{2}}}\right)(y)
\end{aligned}
$$

Similarly,

$$
\left(q_{\mathcal{C}_{1}} \cdot e^{i \nu_{\mathcal{C}_{1}}} \cap q_{\mathcal{C}_{2}} \cdot e^{i \nu_{\mathcal{C}_{2}}}\right)(x y) \geq\left(q_{\mathcal{C}_{1}} \cdot e^{i \nu_{\mathcal{C}_{1}}} \cap q_{\mathcal{C}_{2}} \cdot e^{i \nu_{\mathcal{C}_{2}}}\right)(y) .
$$

And

$$
\begin{aligned}
\left(r_{\mathcal{C}_{1}} \cdot e^{\left.i \omega_{\mathcal{C}_{1}} \cup r_{\mathcal{C}_{2}} \cdot e^{i \omega_{\mathcal{C}_{2}}}\right)(x y)}\right. & =\max \left\{r_{\mathcal{C}_{1}}(x y) \cdot e^{i \omega_{\mathcal{C}_{1}}(x y)}, r_{\mathcal{C}_{2}}(x y) \cdot e^{i \omega_{\mathcal{C}_{2}}(x y)}\right\} \\
& \leq \max \left\{r_{\mathcal{C}_{1}}(y) \cdot e^{i \omega_{\mathcal{C}_{1}}(y)}, r_{\mathcal{C}_{2}}(y) \cdot e^{i \omega_{\mathcal{C}_{2}}(y)}\right\} \\
& =\left(r_{\mathcal{C}_{1}} \cdot e^{i \omega_{\mathcal{C}_{1}}} \cup r_{\mathcal{C}_{2}} \cdot e^{i \omega_{\mathcal{C}_{2}}}\right)(y)
\end{aligned}
$$

Thus $\mathcal{C}_{1} \cap \mathcal{C}_{2}$ is a complex neutrosophic left ideal of semigroup $\mathcal{S}$.

The intersection of complex neutrosophic right ideal can be proved in a similar manner.

Proposition 5.6. If $\mathcal{S}$ be a semigroup. Then the following properties are hold.

(1) The union of two complex neutrosophic subsemigroups of $\mathcal{S}$ is a complex neutrosophic subsemigroup of $\mathcal{S}$.

(2) The union of two complex neutrosophic left (resp., right) ideals of $\mathcal{S}$ is a complex neutrosophic left (resp., right) ideal of $\mathcal{S}$.

Proof: Let

$$
\mathcal{C}_{1}=\left\langle\mathcal{C}_{1 T}=p_{\mathcal{C}_{1}} \cdot e^{i \mu_{\mathcal{C}_{1}}}, \mathcal{C}_{1 I}=q_{\mathcal{C}_{1}} \cdot e^{i \nu_{\mathcal{C}_{1}}}, \mathcal{C}_{1 F}=r_{\mathcal{C}_{1}} \cdot e^{i \omega_{\mathcal{C}_{1}}}\right\rangle
$$


and

$$
\mathcal{C}_{2}=\left\langle\mathcal{C}_{2 T}=p_{\mathcal{C}_{2}} \cdot e^{i \mu_{\mathcal{C}_{2}}}, \mathcal{C}_{2 I}=q_{\mathcal{C}_{2}} \cdot e^{i \nu_{\mathcal{C}_{2}}}, \mathcal{C}_{2 F}=r_{\mathcal{C}_{2}} \cdot e^{i \omega_{\mathcal{C}_{2}}}\right\rangle
$$

be any two complex neutrosophic subsemigroups of $\mathcal{S}$. Let $x, y \in \mathcal{S}$. Then

$$
\begin{aligned}
\left(p_{\mathcal{C}_{1}} \cdot e^{i \mu_{\mathcal{C}_{1}}} \cup p_{\mathcal{C}_{2}} \cdot e^{i \mu_{\mathcal{C}_{2}}}\right)(x y)= & \max \left\{p_{\mathcal{C}_{1}}(x y) \cdot e^{i \mu_{\mathcal{C}_{1}}(x y)}, p_{\mathcal{C}_{2}}(x y) \cdot e^{i \mu_{\mathcal{C}_{2}}(x y)}\right\} \\
\geq & \max \left\{\min \left\{p_{\mathcal{C}_{1}}(x) \cdot e^{i \mu_{\mathcal{C}_{1}}(x)}, p_{\mathcal{C}_{1}}(y) \cdot e^{i \mu_{\mathcal{C}_{1}}(y)}\right\}\right. \\
& \left.\min \left\{p_{\mathcal{C}_{2}}(x) \cdot e^{i \mu_{\mathcal{C}_{2}}(x)}, p_{\mathcal{C}_{2}}(y) \cdot e^{i \mu_{\mathcal{C}_{2}}(y)}\right\}\right\} \\
= & p_{\mathcal{C}_{1}}(x) \cdot e^{i \mu_{\mathcal{C}_{1}}(x)} \wedge p_{\mathcal{C}_{1}}(y) \cdot e^{i \mu_{\mathcal{C}_{1}}(y)} \vee \\
& p_{\mathcal{C}_{2}}(x) \cdot e^{i \mu_{\mathcal{C}_{2}}(x)} \wedge p_{\mathcal{C}_{2}}(y) \cdot e^{i \mu_{\mathcal{C}_{2}}(y)} \\
= & p_{\mathcal{C}_{1}}(x) \cdot e^{i \mu_{\mathcal{C}_{1}}(x)} \vee p_{\mathcal{C}_{2}}(x) \cdot e^{i \mu_{\mathcal{C}_{2}}(x)} \wedge \\
& p_{\mathcal{C}_{1}}(y) \cdot e^{i \mu_{\mathcal{C}_{1}}(y)} \vee p_{\mathcal{C}_{2}}(y) \cdot e^{i \mu_{\mathcal{C}_{2}}(y)} \\
= & \min \left\{\left(p_{\mathcal{C}_{1}} \cdot e^{i \mu_{\mathcal{C}_{1}}} \cup p_{\mathcal{C}_{2}} \cdot e^{i \mu_{\mathcal{C}_{2}}}\right)(x)\right. \\
& \left.\left(p_{\mathcal{C}_{1}} \cdot e^{i \mu_{\mathcal{C}_{1}}} \cup p_{\mathcal{C}_{2}} \cdot e^{i \mu_{\mathcal{C}_{2}}}\right)(y)\right\}
\end{aligned}
$$

Similarly,

$$
\begin{aligned}
\left(q_{\mathcal{C}_{1}} \cdot e^{i \nu_{\mathcal{C}_{1}}} \cup q_{\mathcal{C}_{2}} \cdot e^{i \nu_{\mathcal{C}_{2}}}\right)(x y) \geq & \min \left\{\left(q_{\mathcal{C}_{1}} \cdot e^{i \nu_{\mathcal{C}_{1}}} \cup q_{\mathcal{C}_{2}} \cdot e^{i \nu_{\mathcal{C}_{2}}}\right)(x),\right. \\
& \left.\left(q_{\mathcal{C}_{1}} \cdot e^{i \nu_{\mathcal{C}_{1}}} \cup q_{\mathcal{C}_{2}} \cdot e^{i \nu_{\mathcal{C}_{2}}}\right)(y)\right\}
\end{aligned}
$$

And

$$
\begin{aligned}
& \left(r_{\mathcal{C}_{1}} \cdot e^{i \omega_{\mathcal{C}_{1}}} \cap r_{\mathcal{C}_{2}} \cdot e^{i \omega_{\mathcal{C}_{2}}}\right)(x y)=\min \left\{r_{\mathcal{C}_{1}}(x y) \cdot e^{i \omega_{\mathcal{C}_{1}}(x y)}, r_{\mathcal{C}_{2}}(x y) \cdot e^{i \omega_{\mathcal{C}_{2}}(x y)}\right\} \\
& \leq \min \left\{\max \left\{r_{\mathcal{C}_{1}}(x) \cdot e^{i \omega_{\mathcal{C}_{1}}(x)}, r_{\mathcal{C}_{1}}(y) \cdot e^{i \omega_{\mathcal{C}_{1}}(y)}\right\},\right. \\
& \left.\max \left\{r_{\mathcal{C}_{2}}(x) \cdot e^{i \omega_{\mathcal{C}_{2}}(x)}, r_{\mathcal{C}_{2}}(y) \cdot e^{i \omega_{\mathcal{C}_{2}}(y)}\right\}\right\} \\
& =r_{\mathcal{C}_{1}}(x) \cdot e^{i \omega_{\mathcal{C}_{1}}(x)} \vee r_{\mathcal{C}_{1}}(y) \cdot e^{i \omega_{\mathcal{C}_{1}}(y)} \wedge \\
& r_{\mathcal{C}_{2}}(x) \cdot e^{i \omega_{\mathcal{C}_{2}}(x)} \vee r_{\mathcal{C}_{2}}(y) \cdot e^{i \omega_{\mathcal{C}_{2}}(y)} \\
& =r_{\mathcal{C}_{1}}(x) \cdot e^{i \omega_{\mathcal{C}_{1}}(x)} \wedge r_{\mathcal{C}_{2}}(x) \cdot e^{i \omega_{\mathcal{C}_{2}}(x)} \vee \\
& r_{\mathcal{C}_{1}}(y) \cdot e^{i \omega_{\mathcal{C}_{1}}(y)} \wedge r_{\mathcal{C}_{2}}(y) \cdot e^{i \omega_{\mathcal{C}_{2}}(y)} \\
& =\max \left\{\left(r_{\mathcal{C}_{1}} \cdot e^{i \omega_{\mathcal{C}_{1}}} \cap r_{\mathcal{C}_{2}} \cdot e^{i \omega_{\mathcal{C}_{2}}}\right)(x),\right. \\
& \left.\left(r_{\mathcal{C}_{1}} \cdot e^{i \omega_{\mathcal{C}_{1}}} \cap r_{\mathcal{C}_{2}} \cdot e^{i \omega_{\mathcal{C}_{2}}}\right)(y)\right\}
\end{aligned}
$$

Therefore, $\mathcal{C}_{1} \cup \mathcal{C}_{2}$ is a complex neutrosophic subsemigroup of $\mathcal{S}$. 
(2) Let $\mathcal{C}_{1}$ and $\mathcal{C}_{2}$ be any two complex neutrosophic left ideals of semigroup $\mathcal{S}$, and $x, y \in \mathcal{S}$. Then

$$
\begin{aligned}
\left(p_{\mathcal{C}_{1}} \cdot e^{i \mu_{\mathcal{C}_{1}}} \cup p_{\mathcal{C}_{2}} \cdot e^{i \mu_{\mathcal{C}_{2}}}\right)(x y) & =\max \left\{p_{\mathcal{C}_{1}}(x y) \cdot e^{i \mu_{\mathcal{C}_{1}}(x y)}, p_{\mathcal{C}_{2}}(x y) \cdot e^{i \mu_{\mathcal{C}_{2}}(x y)}\right\} \\
& \geq \max \left\{p_{\mathcal{C}_{1}}(y) \cdot e^{i \mu_{\mathcal{C}_{1}}(y)}, p_{\mathcal{C}_{2}}(y) \cdot e^{i \mu_{\mathcal{C}_{2}}(y)}\right\} \\
& =\left(p_{\mathcal{C}_{1}} \cdot e^{i \mu_{\mathcal{C}_{1}}} \cup p_{\mathcal{C}_{2}} \cdot e^{i \mu_{\mathcal{C}_{2}}}\right)(y)
\end{aligned}
$$

Similarly,

$$
\left(q_{\mathcal{C}_{1}} \cdot e^{i \nu_{\mathcal{C}_{1}}} \cup q_{\mathcal{C}_{2}} \cdot e^{i \nu_{\mathcal{C}_{2}}}\right)(x y) \geq\left(q_{\mathcal{C}_{1}} \cdot e^{i \nu_{\mathcal{C}_{1}}} \cup q_{\mathcal{C}_{2}} \cdot e^{i \nu_{\mathcal{C}_{2}}}\right)(y) .
$$

And

$$
\begin{aligned}
\left(r_{\mathcal{C}_{1}} \cdot e^{i \omega_{\mathcal{C}_{1}}} \cap r_{\mathcal{C}_{2}} \cdot e^{i \omega_{\mathcal{C}_{2}}}\right)(x y) & =\min \left\{r_{\mathcal{C}_{1}}(x y) \cdot e^{i \omega_{\mathcal{C}_{1}}(x y)}, r_{\mathcal{C}_{2}}(x y) \cdot e^{i \omega_{\mathcal{C}_{2}}(x y)}\right\} \\
& \leq \min \left\{r_{\mathcal{C}_{1}}(y) \cdot e^{i \omega_{\mathcal{C}_{1}}(y)}, r_{\mathcal{C}_{2}}(y) \cdot e^{i \omega_{\mathcal{C}_{2}}(y)}\right\} \\
& =\left(r_{\mathcal{C}_{1}} \cdot e^{i \omega_{\mathcal{C}_{1}}} \cap r_{\mathcal{C}_{2}} \cdot e^{i \omega_{\mathcal{C}_{2}}}\right)(y) .
\end{aligned}
$$

Thus $\mathcal{C}_{1} \cup \mathcal{C}_{2}$ is a complex neutrosophic left ideal of semigroup $\mathcal{S}$.

The union of complex neutrosophic right ideal can be proved in a similar manner.

Theorem 5.7. If $\mathcal{C}_{1}$ and $\mathcal{C}_{2}$ be a complex neutrosophic right and left ideals of a semigroup $\mathcal{S}$, respectively. Then $\mathcal{C}_{1} \otimes \mathcal{C}_{2} \subseteq \mathcal{C}_{1} \cap \mathcal{C}_{2}$.

Proof: Let $\mathcal{C}_{1}$ is complex neutrosophic right ideal and $\mathcal{C}_{2}$ is any complex left neutrosophic ideal of $\mathcal{S}$. Then by Proposition 5.3 and Proposition 5.4 we have $\mathcal{C}_{1} \otimes \mathcal{C}_{2} \subseteq \mathcal{C}_{1} \otimes \mathcal{S} \subseteq \mathcal{C}_{1}$ and $\mathcal{C}_{1} \otimes \mathcal{C}_{2} \subseteq \mathcal{S} \otimes \mathcal{C}_{2} \subseteq \mathcal{C}_{2}$. Hence $\mathcal{C}_{1} \otimes \mathcal{C}_{2} \subseteq \mathcal{C}_{1} \cap \mathcal{C}_{2}$.

Theorem 5.8. If $\mathcal{S}$ is regular semigroup, then $\mathcal{C}_{1} \otimes \mathcal{C}_{2}=\mathcal{C}_{1} \cap \mathcal{C}_{2}$ for every complex neutrosophic right ideal $\mathcal{C}_{1}=\left\langle p_{\mathcal{C}_{1}} \cdot e^{i \mu_{\mathcal{C}_{1}}}, q_{\mathcal{C}_{1}} \cdot e^{i \nu_{\mathcal{C}_{1}}}, r_{\mathcal{C}_{1}} \cdot e^{i \omega_{\mathcal{C}_{1}}}\right\rangle$ and every complex neutrosophic left ideal

$\mathcal{C}_{2}=\left\langle p_{\mathcal{C}_{2}} \cdot e^{i \mu_{\mathcal{C}_{2}}}, q_{\mathcal{C}_{2}} \cdot e^{i \nu_{\mathcal{C}_{2}}}, r_{\mathcal{C}_{2}} \cdot e^{i \omega_{\mathcal{C}_{2}}}\right\rangle$ of $\mathcal{S}$.

Proof: Let $\alpha$ be any element of $\mathcal{S}$. Since $\mathcal{S}$ is regular, there exist an element $x \in \mathcal{S}$ such that $\alpha=\alpha x \alpha$. Hence we have

$$
\begin{aligned}
& \left(p_{\mathcal{C}_{1}} \cdot e^{i \mu_{\mathcal{C}_{1}}} \circ p_{\mathcal{C}_{2}} \cdot e^{i \mu_{\mathcal{C}_{2}}}\right)(\alpha)=\sup _{\alpha=y \kappa}\left\{\min \left\{p_{\mathcal{C}_{1}}(y) \cdot e^{i \mu_{\mathcal{C}_{1}}(y)}, p_{\mathcal{C}_{2}}(\kappa) \cdot e^{i \mu_{\mathcal{C}_{2}}(\kappa)}\right\}\right\} \\
& =\sup _{\alpha x \alpha=y \kappa}\left\{\min \left\{p_{\mathcal{C}_{1}}(y) \cdot e^{i \mu_{\mathcal{C}_{1}}(y)}, p_{\mathcal{C}_{2}}(\kappa) \cdot e^{i \mu_{\mathcal{C}_{2}}(\kappa)}\right\}\right\} \\
& \geq \min \left\{p_{\mathcal{C}_{1}}(\alpha x) \cdot e^{i \mu_{\mathcal{C}_{1}}(\alpha x)}, p_{\mathcal{C}_{2}}(\alpha) \cdot e^{i \mu_{\mathcal{C}_{2}}(\alpha)}\right\} \\
& \geq \min \left\{p_{\mathcal{C}_{1}}(\alpha) \cdot e^{i \mu_{\mathcal{C}_{1}}(\alpha)}, p_{\mathcal{C}_{2}}(\alpha) \cdot e^{i \mu_{\mathcal{C}_{2}}(\alpha)}\right\} \\
& =\left(p_{\mathcal{C}_{1}} \cdot e^{i \mu_{\mathcal{C}_{1}}} \cap p_{\mathcal{C}_{2}} \cdot e^{i \mu_{\mathcal{C}_{2}}}\right)(\alpha) .
\end{aligned}
$$


Similarly,

$$
\left(q_{\mathcal{C}_{1}} \cdot e^{i \nu_{\mathcal{C}_{1}}} \circ q_{\mathcal{C}_{2}} \cdot e^{i \nu_{\mathcal{C}_{2}}}\right)(\alpha) \geq\left(q_{\mathcal{C}_{1}} \cdot e^{i \nu_{\mathcal{C}_{1}}} \cap q_{\mathcal{C}_{2}} \cdot e^{i \nu_{\mathcal{C}_{2}}}\right)(\alpha)
$$

And

$$
\begin{aligned}
& \left(r_{\mathcal{C}_{1}} \cdot e^{i \omega_{\mathcal{C}_{1}}} \circ r_{\mathcal{C}_{2}} \cdot e^{i \omega_{\mathcal{C}_{2}}}\right)(\alpha)=\inf _{\alpha=y \kappa}\left\{\max \left\{r_{\mathcal{C}_{1}}(y) \cdot e^{i \omega_{\mathcal{C}_{1}}(y)}, r_{\mathcal{C}_{2}}(\kappa) \cdot e^{i \omega_{\mathcal{C}_{2}}(\kappa)}\right\}\right\} \\
& =\inf _{\alpha x \alpha=y \kappa}\left\{\max \left\{r_{\mathcal{C}_{1}}(y) \cdot e^{i \omega_{\mathcal{C}_{1}}(y)}, r_{\mathcal{C}_{2}}(\kappa) \cdot e^{i \omega_{\mathcal{C}_{2}}(\kappa)}\right\}\right\} \\
& \leq \max \left\{r_{\mathcal{C}_{1}}(\alpha x) \cdot e^{i \omega_{\mathcal{C}_{1}}(\alpha x)}, r_{\mathcal{C}_{2}}(\alpha) \cdot e^{i \omega_{\mathcal{C}_{2}}(\alpha)}\right\} \\
& \leq \max \left\{r_{\mathcal{C}_{1}}(\alpha) \cdot e^{i \omega_{\mathcal{C}_{1}}(\alpha)}, r_{\mathcal{C}_{2}}(\alpha) \cdot e^{i \omega_{\mathcal{C}_{2}}(\alpha)}\right\} \\
& =\left(r_{\mathcal{C}_{1}} \cdot e^{i \omega_{\mathcal{C}_{1}}} \cup r_{\mathcal{C}_{2}} \cdot e^{i \omega_{\mathcal{C}_{2}}}\right)(\alpha) \text {. }
\end{aligned}
$$

So $\mathcal{C}_{1} \otimes \mathcal{C}_{2} \supseteq \mathcal{C}_{1} \cap \mathcal{C}_{2}$, and $\mathcal{C}_{1} \otimes \mathcal{C}_{2} \subseteq \mathcal{C}_{1} \cap \mathcal{C}_{2}$ is true from Theorem 5.7. Hence $\mathcal{C}_{1} \otimes \mathcal{C}_{2}=\mathcal{C}_{1} \cap \mathcal{C}_{2}$.

Theorem 5.9. For a non-empty subset $H$ of a semigroup $\mathcal{S}$. We have

(1) $H$ is a subsemigroup of $\mathcal{S}$ if and only if the characteristic complex neutrosophic set $C_{H}=\left\langle T_{C_{H}}, I_{C_{H}}, F_{C_{H}}\right\rangle$ of $H$ in $\mathcal{S}$ is a complex neutrosophic subsemigroup of $\mathcal{S}$.

(2) $H$ is a left (right) ideal of $\mathcal{S}$ if and only if the characteristic complex neutrosophic set $C_{H}=$ $\left\langle T_{C_{H}}, I_{C_{H}}, F_{C_{H}}\right\rangle$ of $H$ in $\mathcal{S}$ is a complex neutrosophic left (resp., right) ideal of $\mathcal{S}$.

Proof: Straightforward.

Theorem 5.10. For every complex neutrosophic right ideal $\mathcal{C}_{1}=\left\langle T_{\mathcal{C}_{1}}, I_{\mathcal{C}_{1}}, F_{\mathcal{C}_{1}}\right\rangle$ and every complex neutrosophic left ideal $\mathcal{C}_{2}=\left\langle T_{\mathcal{C}_{2}}, I_{\mathcal{C}_{2}}, F_{\mathcal{C}_{2}}\right\rangle$ of a semigroup $\mathcal{S}$, if $\mathcal{C}_{1} \otimes \mathcal{C}_{2}=\mathcal{C}_{1} \cap \mathcal{C}_{2}$, then $\mathcal{S}$ is regular.

Proof: Assume that $\mathcal{C}_{1} \otimes \mathcal{C}_{2}=\mathcal{C}_{1} \cap \mathcal{C}_{2}$ for every complex neutrosophic right ideal $\mathcal{C}_{1}=\left\langle T_{\mathcal{C}_{1}}, I_{\mathcal{C}_{1}}, F_{\mathcal{C}_{1}}\right\rangle$ and every complex neutrosophic left ideal $\mathcal{C}_{2}=\left\langle T_{\mathcal{C}_{2}}, I_{\mathcal{C}_{2}}, F_{\mathcal{C}_{2}}\right\rangle$ of a semigroup $\mathcal{S}$. Let $\mathcal{R}$ and $\mathcal{L}$ be any right and left ideal of $\mathcal{S}$, respectively. In order to see that $\mathcal{R} \cap \mathcal{L} \subseteq \mathcal{R} \mathcal{L}$ holds. Let $\alpha$ be any element of $\mathcal{R} \cap \mathcal{L}$, then the characteristic complex neutrosophic sets $C_{\mathcal{R}}=\left\langle T_{C_{\mathcal{R}}}, I_{C_{\mathcal{R}}}, F_{C_{\mathcal{R}}}\right\rangle$ and $C_{\mathcal{L}}=\left\langle T_{C_{\mathcal{L}}}, I_{C_{\mathcal{L}}}, F_{C_{\mathcal{L}}}\right\rangle$ are a complex neutrosophic right ideal and a complex neutrosophic left ideal of $\mathcal{S}$, respectively, by Theorem 5.9.

It follows from the hypothesis and proposition 5.2 , that is

$$
\begin{aligned}
T_{C_{\mathcal{R} \mathcal{L}}}(\alpha) & =\left(T_{C_{\mathcal{R}}} \circ T_{C_{\mathcal{L}}}\right)(\alpha)=\left(T_{C_{\mathcal{R}}} \cap T_{C_{\mathcal{L}}}\right)(\alpha) \\
& =T_{C_{\mathcal{R} \cap \mathcal{L}}}(\alpha)=1 . e^{i 2 \pi} \\
I_{C_{\mathcal{R} \mathcal{L}}}(\alpha) & =\left(I_{C_{\mathcal{R}}} \circ I_{C_{\mathcal{L}}}\right)(\alpha)=\left(I_{\left.C_{\mathcal{R}} \cap I_{C_{\mathcal{L}}}\right)(\alpha)}\right. \\
& =I_{C_{\mathcal{R} \cap \mathcal{L}}}(\alpha)=1 . e^{i 2 \pi}
\end{aligned}
$$


and

$$
\begin{aligned}
F_{C_{\mathcal{R} \mathcal{L}}}(\alpha) & =\left(F_{C_{\mathcal{R}}} \circ F_{C_{\mathcal{L}}}\right)(\alpha)=\left(F_{C_{\mathcal{R}}} \cup F_{C_{\mathcal{L}}}\right)(\alpha) \\
& =F_{C_{\mathcal{R} \cup \mathcal{L}}}(\alpha)=0 .
\end{aligned}
$$

So that $\alpha \in \mathcal{R L}$. Thus $\mathcal{R} \cap \mathcal{L} \subseteq \mathcal{R} \mathcal{L}$. Since the inclusion in the other direction always holds, we obtain that $\mathcal{R} \cap \mathcal{L} \subseteq \mathcal{R} \mathcal{L}$. It follows from Lemma 2.1, that $\mathcal{S}$ is regular.

\section{REFERENCES}

[1] Zadeh, L. A. Fuzzy Sets. Inf. Control, 8 (1965), 338-353.

[2] Atanassov, K. T. Intuitionistic Fuzzy Sets, Fuzzy Sets Syst. 20 (1986), 87-96.

[3] Kuroki, N. Fuzzy Bi-ideals in Semigroups. Comment. Math. Univ. St. Pauli, 27 (1979), 17-21.

[4] Wang, H. et al. Single Valued Neutrosophic Sets. Proc of 10th Int Conf on Fuzzy Theory and Technology, Salt Lake City, Utah (2005).

[5] Smarandache, F. A Unifying Field in Logics. Neutrosophy: Neutrosophic Probability, Set and logic, Rehoboth; American Research Press (1999).

[6] Buckley, J. J. Fuzzy Complex Numbers. Fuzzy Sets Syst. 33 (1989), 333-345.

[7] Nguyen, H. T. Kandel, A. and Kreinovich, V. Complex Fuzzy Sets. Towards New Foundations, IEEE. (2000), 7803-5877.

[8] Ramot, D. Milo, R. Friedman, M. Kandel, A. Complex fuzzy sets. IEEE Trans. Fuzzy Syst. 10 (2002), 171-186.

[9] Sveinn, R. J. Haskoli Islands, Verkfraeoideild. Complex Fuzzy Sets. IEEE Trans. Fuzzy Syst. 10 (2002), 171 - 186.

[10] Zhang, G. Dillon, T. S. Cai, K. Y. Ma, J. and Lu, J. Operation Properties and $\delta$-Equalities of Complex Fuzzy Sets. Int. J. Approx. Reason. 50 (2009), 1227-1249.

[11] Jun, Y. B. and Khan, A. Cubic Ideals in Semigroups. Honam Math. J. 35 (2013), 607-623.

[12] Abd Ulazeez, M. Alkouri, S. and Salleh, A. R. Complex Intuitionistic Fuzzy Sets. International Conference on Fundamental and Applied Sciences, AIP Conf. Proc. 1482 (2012), 464-470.

[13] Abd Ulazeez, M. Alkouri, S. and Salleh, A. R. Complex Atanassov's Intuitionistic Fuzzy Relation. Hindawi Publishing Corporation Abstr. Appl. Anal. 2013 (2013), Article ID 287382, 18pages.

[14] Ali, M and Smarandache, F. Complex Neutrosophic Set. Neural. Comput. Applic. 28 (2017), 18171834.

[15] Cetkin, V. and Aygun, H. An Approach to Neutrosophic Subgroup and its Fundamental Properties. J. Intell. Fuzzy Syst. 29 (2015), 1941-1947.

[16] Iseki, K. A characterization of regular semigroups, Proc. Japan Acad. 32 (1965), 676-677.

[17] Turksen, I. B. Interval-Valued Fuzzy Sets Based on Normal Forms. Fuzzy Sets Syst. 20 (1986), 191-210. 